\title{
Master integrals for inclusive weak decays of heavy flavors at next-to-leading order
}

\author{
Th. Mannel, D. Moreno ${ }^{\circ}$, and A. A. Pivovarov ${ }^{(1)}$ \\ Center for Particle Physics Siegen, Theoretische Physik 1, Universität Siegen, 57068 Siegen, Germany
}

(Received 24 August 2021; accepted 2 December 2021; published 27 December 2021)

\begin{abstract}
We present analytical results for master integrals emerging in the computation of differential rates for inclusive weak decays of heavy flavors at next-to-leading order (NLO) in QCD. As an immediate physical application, these master integrals allow for a calculation of the spectra of the leptonic invariant mass in inclusive semileptonic decays in the framework of the heavy quark expansion, including the NLO QCD corrections to power-suppressed terms.
\end{abstract}

DOI: 10.1103/PhysRevD.104.114035

\section{INTRODUCTION}

The heavy quark expansion (HQE) [1-5] provides a solid framework for the computation of observables for hadrons containing a heavy quark. For this reason it has become the standard tool to analyze inclusive heavy hadron decays, which has been refined over the past three decades by detailed calculations of higher order perturbative contributions, as well as by inclusion of nonperturbative contributions. The techniques for the computation of inclusive semileptonic widths and distributions have been developed long ago [6-9]. However, the fully differential width at order $\alpha_{s}$ was obtained only 15 years ago [10-12], including double and triple differential rates and spectral moments, some of which could be computed even analytically.

From the phenomenological side, inclusive decays are of great interest for the precise extraction of CabibboKobayashi-Maskawa (CKM) matrix elements [13,14], which play a central role for testing the flavor sector of the Standard Model of particle physics (SM) and for the search for flavor and $C P$ violation beyond the SM.

In this paper we describe a useful setup for the computation of $\alpha_{s}$ corrections to differential rates in some specific kinematic invariants. In particular, this setup is designed such that it can easily be extended to the calculation of next-to-leading order (NLO) QCD corrections to power-suppressed terms within the HQE. To this end, it opens the road for analytical NLO calculations for differential rates for power-suppressed terms, which is phenomenologically important, since this allows us to implement phase-space cuts in an easy way.

Published by the American Physical Society under the terms of the Creative Commons Attribution 4.0 International license. Further distribution of this work must maintain attribution to the author(s) and the published article's title, journal citation, and DOI. Funded by SCOAP .
The approach makes use of modern tools for the computation of multiloop Feynman integrals, in particular the program LITERED $[15,16]$. That allows one to write the Feynman diagram amplitudes as a combination of master integrals, which are our key ingredients. These are up to two loop integrals with two scales which we compute analytically, meaning in terms of well-known and wellstudied special functions.

Even though inclusive semileptonic decays are the main target for phenomenological applications, the master integrals we compute here can be applied to other inclusive decays, such as nonleptonic or exotic decays.

In particular, our formulation allows us to calculate the total rate with final states with three different masses even for the power-suppressed terms. At leading power the result is known [17], and we use this result as a check of our calculation.

As an application, we compute for the first time the inclusive semileptonic differential width in the lepton pair invariant mass square with massive charm and massless leptons analytically at the leading power to $\mathcal{O}\left(\alpha_{s}\right)$.

We use standard renormalization and dimensional regularization $(D=4-2 \epsilon)$ [18] with anticommuting $\gamma_{5}$ $[19,20]$. Therefore, the Dirac algebra of $\gamma$ matrices usually defined in $D=4$ needs to be extended to $D$-dimensional spacetime [21-24]. The Dirac and Lorentz algebra is manipulated using TRACER [25] and the $\epsilon$ expansion of hypergeometric functions is computed with the help of HYPEXP $[26,27]$.

The paper is organized as follows. In Sec. II we explain the approach used for the computation of the differential decay width. In Sec. III we compute the necessary master integrals. In Sec. IV we apply the results of Sec. III to the computation of the semileptonic width differential in the lepton pair invariant mass square, as well as the total semileptonic width. Finally, we present the results for the $\mathrm{HQE}$ coefficients of the differential and total semileptonic 
decay width in Sec. V. We also give some technical results in Appendix.

\section{FORMULATION OF THE METHOD}

We consider the inclusive decay of a heavy flavored hadron, which is induced by the quark-level transition

$$
Q(M) \rightarrow q(m) p_{1}\left(m_{1}\right) \cdots p_{n}\left(m_{n}\right)
$$

where $Q$ is a heavy quark of mass $M, q$ is a lighter quark of mass $m$, and $p_{i}$ stands for any type of particles with mass $m_{i}$. In typical SM processes the $p_{i}$ are either quarks or leptons, but in fact they can be of any type (fermions, scalars,...); i.e., our results are very general.

Using the optical theorem the decay width of a heavyflavored hadron can be computed from the discontinuity of the forward scattering matrix element, for which the HQE can be employed, yielding a systematic expansion in powers of $\Lambda_{\mathrm{QCD}} / M \ll 1$ :

$$
\begin{aligned}
\Gamma\left(Q \rightarrow q p_{1} \cdots p_{n}\right) & =\frac{1}{M_{H_{Q}}}\left\langle H_{Q}\left(p_{H_{Q}}\right)|\operatorname{Im} \hat{T}| H_{Q}\left(p_{H_{Q}}\right)\right\rangle \\
& =\Gamma^{0}\left(C_{0}\left(\rho, \eta_{i}\right)+\mathcal{O}(1 / M)\right),
\end{aligned}
$$

where $\left|H_{Q}\left(p_{H_{Q}}\right)\right\rangle$ stands for the full hadronic states with four-momentum $p_{H_{Q}}$ and mass $M_{H_{Q}}$, the transition operator $\hat{T}$ is related to the scattering operator $\hat{S}$ by $\hat{S}=1+i \hat{T}$, the normalization factor $\Gamma^{0}$ contains all trivial information such as phase-space factors and dimensionful scales and couplings, and $C_{0}$ is the leading order matching coefficient in the HQE, which depends on the dimensionless parameters $\rho=m^{2} / M^{2}$ and $\eta_{i}=m_{i}^{2} / M^{2}(i=1, \ldots, n)$. Note that we assume that $\rho$ and $\eta_{i}$ are treated as parameters of order unity.

The typical Feynman diagram one faces in the computation of the total width at LO-QCD is the $n$-loop graph shown in Fig. 1 (left). At NLO-QCD, the required Feynman

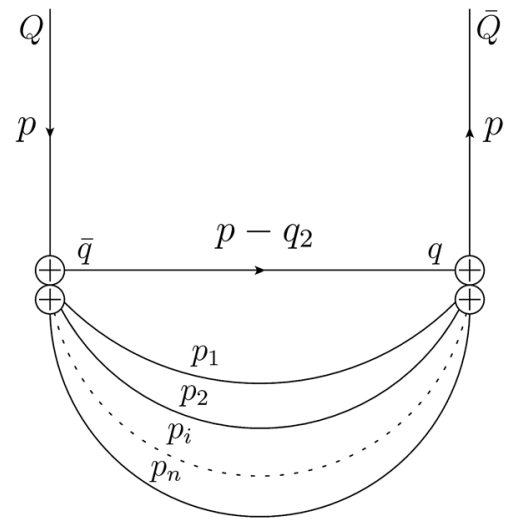

diagrams are $(n+1)$-loop graphs which can be obtained from the one in Fig. 1 (left) by taking one gluon exchange (massless line exchanges) between two massive lines. An example of such diagrams is shown in Fig. 1 (right). To keep the following discussion simple, let us consider colorless particles $p_{i}$, such that the gluon couples only to the quarks $Q$ and $q$. At NLO, this is no restriction of generality.

However, our goal is not the computation of the total width, but the width differential in some kinematic invariant, which we chose to be the invariant mass square of the $n$ $p$-particles. Technically, one can achieve this by factorizing the $n$ massive $p$-particle propagators in a single massive propagator using a dispersion representation

$$
\begin{aligned}
\frac{1}{i} & \int \prod_{j=1}^{n}\left(\frac{d^{D} k_{j}}{(2 \pi)^{D}} \frac{1}{k_{j}^{2}-m_{j}^{2}}\right) \delta^{(4)}\left(q_{2}-\sum_{i=1}^{n} k_{n}\right) \\
= & \int_{\left(\sum_{i=1}^{n} m_{i}\right)^{2}}^{\infty} \frac{\rho_{(n-1) \mathrm{LS}}\left(\chi^{2}, m_{1}, \ldots, m_{n}\right)}{\chi^{2}-q_{2}^{2}-i \eta} d\left(\chi^{2}\right),
\end{aligned}
$$

where $\rho_{(n-1) \text { LS }}$ is the spectral density for the $(n-1)$-loop generalized sunset diagram. For example, at one-loop it is given by Eq. (A2). Note that the $(n-1)$-loop integral in Eq. (3) has become an integral over the mass of an "effective massive propagator" of mass $\chi$, and that the dependence on the $m_{i}$ is completely factorized in the spectral density. This fact is diagrammatically represented in Fig. 2. This representation serves as a powerful tool for both, the computation of the total width and the computation of the decay width differential in the $n p$-particle invariant mass square $q_{2}^{2}=\left(\sum_{i=1}^{n} k_{i}\right)^{2}$. The $(n-1)$-loop in Eq. (3) is always cut, so $q_{2}^{2}$ is forced to be on-shell $q_{2}^{2}=\chi^{2}$, corresponding to the $q_{2}^{2}$ pole on the right-hand side of Eq. (3). Therefore, the integrand is differential in the $n$ p-particle invariant mass square $\chi^{2}$.

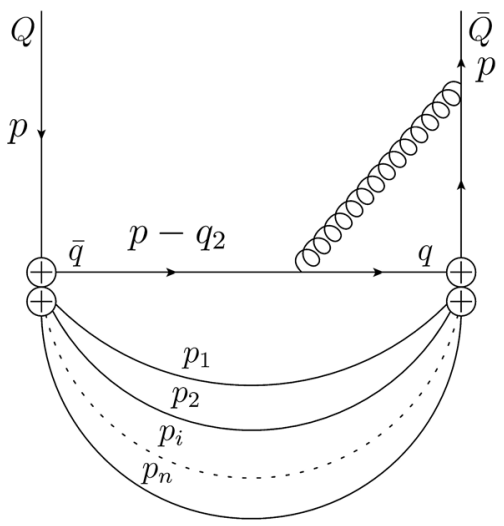

FIG. 1. Examples of Feynman diagrams entering in the calculation of the coefficient of the total width $C_{0}$ at LO-QCD (left) and NLOQCD (right). 

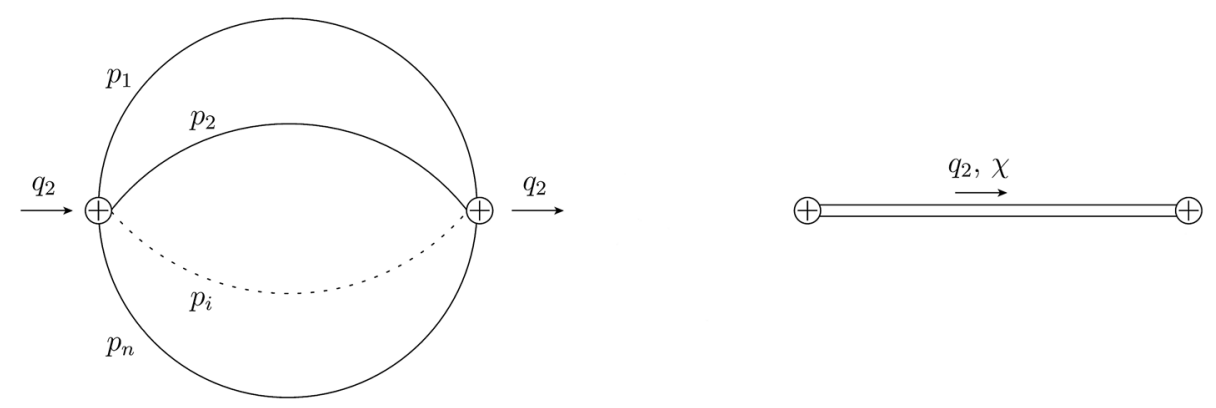

FIG. 2. Factorization of the $n$-loop sunset with all massive lines (left) in a single "effective massive propagator" with spectral density, mass $\chi$, and integration over the $n p$-particle invariant mass square (right).

In this setup, the decay width can be written as

$$
\begin{aligned}
\Gamma(Q & \left.\rightarrow q p_{1} \cdots p_{n}\right) \\
& =\Gamma^{0} \int_{\left(\sum_{i=1}^{n} \sqrt{\eta_{i}}\right)^{2}}^{(1-\sqrt{\rho})^{2}} d r \rho_{s}\left(r, \eta_{i}\right)\left(\mathcal{C}_{0}(r, \rho)+\mathcal{O}(1 / M)\right) \\
& \equiv \int_{\left(\sum_{i=1}^{n} \sqrt{\eta_{i}}\right)^{2}}^{(1-\sqrt{\rho})^{2}} d r \frac{d \Gamma\left(r, \rho, \eta_{i}\right)}{d r}
\end{aligned}
$$

where $r=\chi^{2} / M^{2}$ is the dimensionless invariant mass square of the $n p$-particles, $\rho_{s}$ is the spectral density, which depends on the structure of the interactions, and $\mathcal{C}_{0}$ is the leading matching coefficient in the HQE of the differential width $d \Gamma / d r=\Gamma^{0} \rho_{s} \mathcal{C}_{0}$.

Note that, whereas the computation of the coefficient of the total width requires computing $n$-loop and $(n+1)$-loop diagrams with scales $\left(\rho, \eta_{i}\right)$ at LO-QCD and at NLO-QCD, respectively (see Fig. 1), the computation of the coefficient of the differential width requires computing one-loop and two-loop diagrams with two scales $(r, \rho)$ at LO-QCD and at NLO-QCD, respectively (see Fig. 3). However, for the complete determination of the differential width one has to deal with $(n-1)$-loop integrals with scales $\left(\eta_{i}\right)$ to obtain the spectral density $\rho_{s}$.

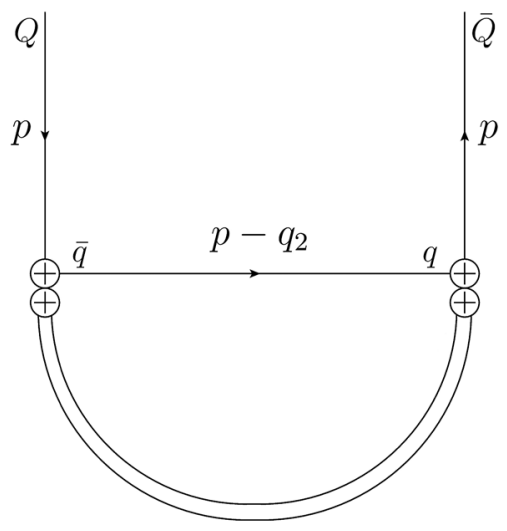

For the computation, we use LITERED to write the amplitudes to a combination of a small set of master integrals. The main task of this paper, which we address in Secs. III B and III C, is the analytical computation of the necessary master integrals for the determination of $\mathcal{C}_{0}(r, \rho)$ at NLO in $\alpha_{s}$. That requires dealing with up to two-loop integrals with two scales $r$ and $\rho$. These master integrals are universal to any diagrams of the type shown in Fig. 1. As a check, in Secs. III D and III E, we independently compute the master integrals in the case where the quark $q$ is massless $(m=0)$ and compare to the massive case in the limit $\rho \rightarrow 0$. From now on, we will refer to the calculation with $m \neq 0$ as the massive case and the calculation with $m=0$ as the massless case.

The coefficient of the differential width is related to the coefficient of the total width through

$$
C_{0}\left(\rho, \eta_{i}\right)=\int_{\left(\sum_{i=1}^{n} \sqrt{\eta_{i}}\right)^{2}}^{(1-\sqrt{\rho})^{2}} d r \rho_{s}\left(r, \eta_{i}\right) \mathcal{C}_{0}(r, \rho)
$$

Finally, we emphasize that the differential rate is of great importance since it allows us to implement cuts on the moments which are unavoidable for the comparison with the experiment. The (not yet normalized) moments with a lower cut $r_{\min }$ and upper cut $r_{\max }$ are defined as

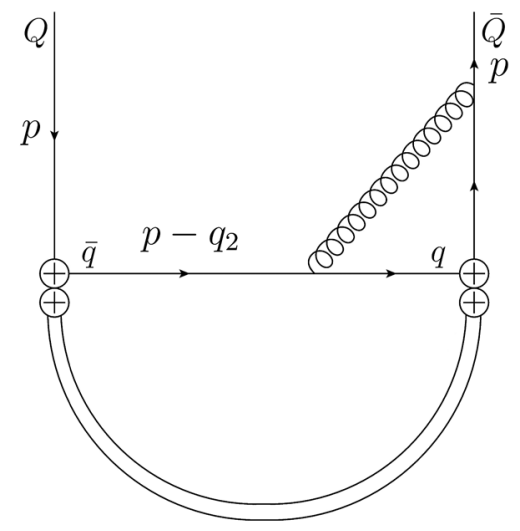

FIG. 3. Examples of Feynman diagrams entering in the calculation of the coefficient of the differential width $\mathcal{C}_{0}$ at LO-QCD (left) and NLO-QCD (right). 


$$
M_{n}\left(\rho, \eta_{i}, r_{\min }, r_{\max }\right)=\int_{r_{\min }}^{r_{\max }} d r r^{n} \frac{d \Gamma\left(r, \rho, \eta_{i}\right)}{d r},
$$

with $\left(\sum_{i=1}^{n} \sqrt{\eta_{i}}\right)^{2}<r_{\min }<r_{\max }<(1-\sqrt{\rho})^{2}$. Obviously, having the expression for the differential width is more powerful than having particular moments of the distribution, since it is more general.

Note that the coefficient of the differential width can always be computed analytically. If $\rho_{s}$ can be computed analytically, then the differential width is known analytically as well, and the final integration over $r$ to obtain the total width or moments (with or without cuts) can always be performed, at least numerically. Numerical evaluation, if reliable, is practical for comparison to experimental data (see e.g., [28] where four-loop integrals with masses have been computed).

Let us stress that the master integrals computed in this paper may have several physical applications. They can be used for the computation of the differential and total semileptonic and nonleptonic decay widths with any number of different masses. For semileptonic and nonleptonic decays the spectral density can always be obtained analytically. Therefore, the total width and moments can be computed straightforwardly, at least numerically. This also applies to power corrections.

While we will formulate the setup and the calculation in a very general language, the main motivation (and probably also the main application) are semileptonic decays into light massless leptons or $\tau$ leptons. The relevant observables are in this case the cut moments of the leptonic invariant mass spectrum, which can be used to extract the value of $V_{c b}$ from the data on inclusive $b \rightarrow c \ell \bar{\nu}$ transitions. In fact, it has been shown in [29] that observables based on the leptonic invariant mass spectrum depend only on a reduced set of nonperturbative parameters and thus an even more precise determination of $V_{c b}$ becomes possible.

In the case of decays into tau leptons an account for the lepton mass is a must. That can be straightforwardly accounted within our setup and requires the change of the spectral density only, while the quark part is untouched. The mass of the muon can be included as well if it is necessary for future phenomenological analysis.

\section{MASTER INTEGRALS}

In this section, we perform the analytical computation of the master integrals necessary for the determination of the coefficient of the differential width $\mathcal{C}_{0}$. The key ingredient is a specific choice of variables which is introduced in the next section. Based on this we will compute the master integrals for the massive and the massless cases.

\section{A. Choice of variables}

In the massive case, computing the differential width at NLO in $\alpha_{s}$ requires the evaluation of two-loop integrals with three masses (two scales). That makes the problem of an analytic computation of the master integrals rather involved. However, the calculation is facilitated once a proper choice of the variables is made, which is motivated by the physics of the system. It is remarkable that such a choice of variables allows us to compute analytically the master integrals including also the full dependence on $\epsilon$, with only a single exception, where we can give an analytic result only as an $\epsilon$ expansion to the necessary order. However, that is sufficient for the phenomenological applications.

Furthermore, in order to arrive at an analytical expression for the total rate, an integration over the $n p$-particle invariant mass square has to be performed. This step is also greatly facilitated by a proper choice of variables.

The naive choice would be to use $r$ and $\rho$ as the most natural variables, but when trying to compute two-loop sunset type integrals with two massive lines one quickly learns that this choice is not appropriate, since it is not adapted to the physics of the problem. In turn, the analytical dependence on $r$ and $\rho$ is complicated; in fact it is too complicated for any known tool to be able to perform the integration analytically.

Rather we suggest a different set of variables $\left(x_{ \pm}\right)$which is motivated by the zeros of the function which develops the cut and which defines the integration region in the imaginary part of the one-loop topology with two propagators. To see this, it is enough to take the left-hand side of Eq. (A1), introduce standard Feynman parametrization, integrate over the loop-momentum and take the imaginary part. One then gets an integral of the form

$$
\begin{aligned}
\operatorname{Im} & \frac{1}{i} \int \frac{d^{D} k}{(2 \pi)^{D}} \frac{1}{\left(k^{2}-m^{2}\right)\left((p-k)^{2}-\chi^{2}\right)} \\
& \sim \int_{x_{-}}^{x_{+}} d x\left[-\left(x-x_{+}\right)\left(x-x_{-}\right)\right]^{-\epsilon},
\end{aligned}
$$

with $D=4-2 \epsilon$. This has a simple form in terms of the new variables $x_{ \pm}$which are related to $r$ and $\rho$ through

$x_{-}=\frac{1}{2}\left(1-r+\rho-\sqrt{\left(1-(\sqrt{r}-\sqrt{\rho})^{2}\right)\left(1-(\sqrt{r}+\sqrt{\rho})^{2}\right)}\right)$,

$x_{+}=\frac{1}{2}\left(1-r+\rho+\sqrt{\left(1-(\sqrt{r}-\sqrt{\rho})^{2}\right)\left(1-(\sqrt{r}+\sqrt{\rho})^{2}\right)}\right)$.

Note that $x_{ \pm}$are real, $0<x_{-}<x_{+}<1$, and

$$
\begin{gathered}
x_{+}-x_{-}=\sqrt{\left(1-(\sqrt{r}-\sqrt{\rho})^{2}\right)\left(1-(\sqrt{r}+\sqrt{\rho})^{2}\right)}, \\
x_{+}+x_{-}=1-r+\rho,
\end{gathered}
$$

while the Jacobian is determined from the derivatives 


$$
\begin{array}{cl}
\frac{d x_{+}}{d r}=-\frac{x_{+}}{x_{+}-x_{-}}, & \frac{d x_{+}}{d \rho}=-\frac{1-x_{+}}{x_{+}-x_{-}}, \\
\frac{d x_{-}}{d r}=\frac{x_{-}}{x_{+}-x_{-}}, & \frac{d x_{-}}{d \rho}=\frac{1-x_{-}}{x_{+}-x_{-}} .
\end{array}
$$

It is remarkable that the inverse transformation is extremely simple,

$$
\begin{gathered}
\rho=x_{+} x_{-}, \\
r=\left(1-x_{+}\right)\left(1-x_{-}\right),
\end{gathered}
$$

compared to Eqs. (8) and (9). This makes the transformation of an expression in terms of $r$ and $\rho$ to expressions in terms of $x_{ \pm}$very easy, while the reverse is much more complicated. We are now ready to compute the relevant master integrals.
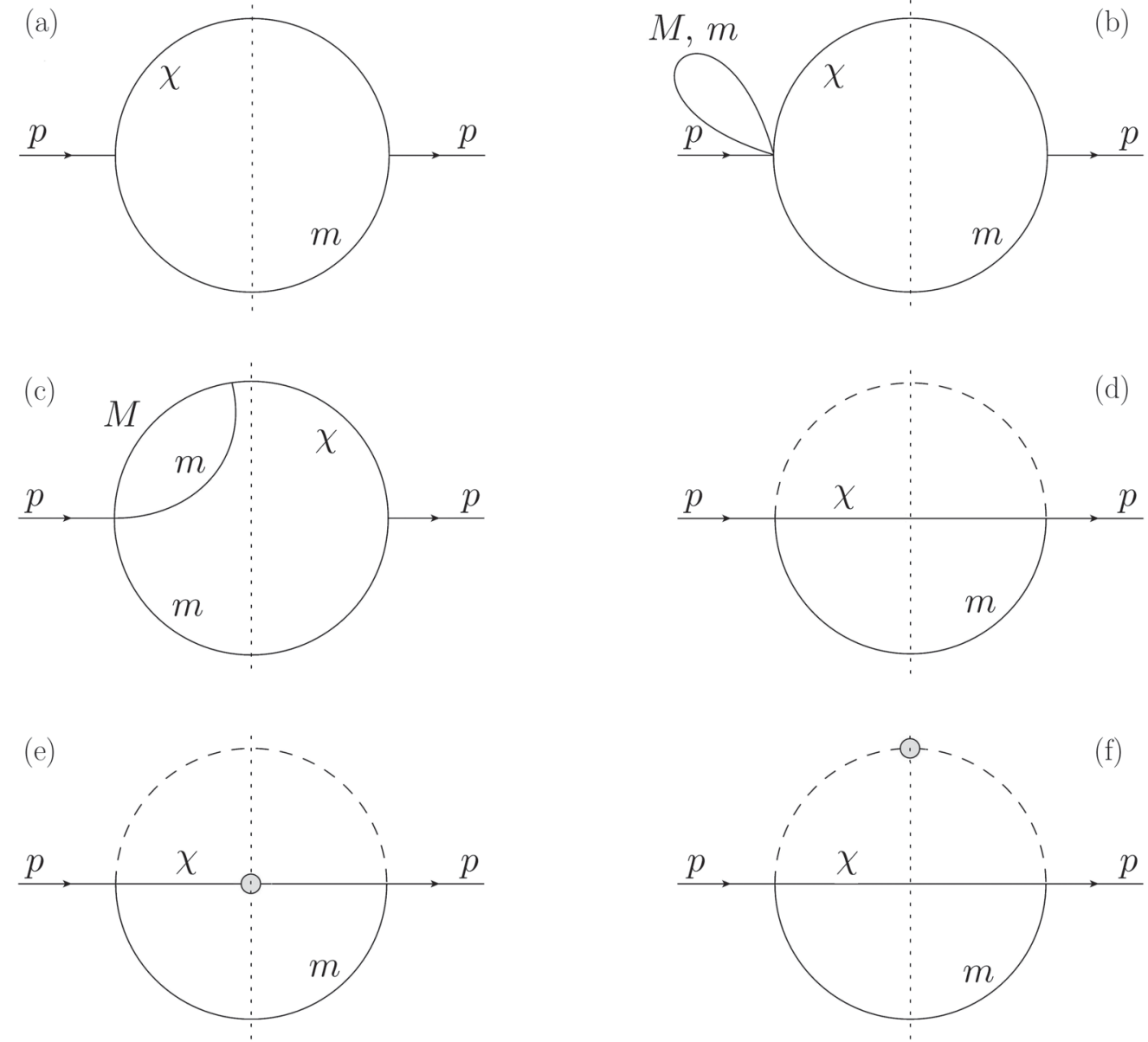

FIG. 4. Master integral topologies stemming from the massive decay channel contributing to the differential decay width up to NLOQCD. Continuous and dashed lines stand for massive and massless propagators, respectively. The gray dot stands for iteration of the corresponding propagator, and the vertical dotted line represents a cut. 


$$
\begin{aligned}
\mathcal{M}(1,1)= & \left(\frac{4 \pi \bar{\mu}^{2}}{M^{2}}\right)^{\epsilon} \frac{1}{16 \pi} \frac{\Gamma(1-\epsilon)}{\Gamma(2-2 \epsilon)}\left(x_{+}-x_{-}\right)^{1-2 \epsilon} \\
& \times \Theta(1-\sqrt{r}-\sqrt{\rho}) .
\end{aligned}
$$

Finally, we have explicitly checked that our results for the master integrals reproduce the ones in the massless case, which are computed in Sec. III D.

\section{NLO-QCD (Massive channel)}

At NLO-QCD the differential decay width for the massive channel can be written as a combination of twoloop five-propagator integrals with three different masses $m, \chi, M$ and on-shell external momenta $p^{2}=M^{2}$ of the form

$$
\begin{aligned}
& \mathcal{J}\left(n_{1}, n_{2}, n_{3}, n_{4}, n_{5}\right) \\
& \quad \equiv \operatorname{Im} \bar{\mu}^{4 \epsilon} \int \frac{d^{D} q_{1}}{(2 \pi)^{D}} \frac{d^{D} q_{2}}{(2 \pi)^{D}} \frac{1}{D_{1}^{n_{1}} D_{2}^{n_{2}} D_{3}^{n_{3}} D_{4}^{n_{4}} D_{5}^{n_{5}}},
\end{aligned}
$$

where

$$
\begin{aligned}
& D_{1}=q_{1}^{2}, \quad D_{2}=q_{2}^{2}-\chi^{2}, \quad D_{3}=\left(p-q_{1}\right)^{2}-M^{2}, \\
& D_{4}=\left(p-q_{2}\right)^{2}-m^{2}, \quad D_{5}=\left(p-q_{1}-q_{2}\right)^{2}-m^{2} .
\end{aligned}
$$

By using LITERED we reduce every integral above as a combination of the following 13 master integrals:

$$
\begin{array}{cc}
\mathcal{J}(0,0,0,1,1), & \mathcal{J}(0,1,0,1,1), \\
\mathcal{J}(0,0,1,0,1), & \mathcal{J}(0,1,1,1,0), \\
\mathcal{J}(0,0,1,1,1), & \mathcal{J}(0,1,1,1,1), \\
\mathcal{J}(0,0,2,1,1), & \mathcal{J}(1,1,0,0,1), \\
\mathcal{J}(0,1,0,0,1), & \mathcal{J}(1,2,0,0,1), \\
\mathcal{J}(0,1,1,0,0), & \mathcal{J}(2,1,0,0,1), \\
\mathcal{J}(0,1,1,0,1) . &
\end{array}
$$

The integrals in the left column are either massive tadpole or two-loop sunset-type diagrams. However, taking the cut procedure (i.e., taking the imaginary part) these diagrams turn out to be zero. This is, on the one hand, due to the fact that tadpole diagrams have no imaginary part and, on the other hand, due to the cut procedure puts the lines on-shell; the diagrams vanish since no phase space is available. We have checked these statements also by explicit calculation.

The master integrals $\mathcal{J}(0,1,0,1,1)$ and $\mathcal{J}(0,1,1,1,0)$ are factorizable into a product of the cut one-loop twopropagator diagram with two massive lines $m$ and $\chi$, computed in Sec. III B, and a closed massive loop with mass $m$ and $M$, respectively. No other cut is possible. The corresponding topologies are shown in Fig. 4(b). They read

$$
\begin{aligned}
\mathcal{J}(0,1,0,1,1)= & \left(\frac{4 \pi \bar{\mu}^{2}}{M^{2}}\right)^{2 \epsilon} \frac{M^{2}}{256 \pi^{3}} \frac{\Gamma(-1+\epsilon) \Gamma(1-\epsilon)}{\Gamma(2-2 \epsilon)} \\
& \times\left(x_{+} x_{-}\right)^{1-\epsilon}\left(x_{+}-x_{-}\right)^{1-2 \epsilon} \\
& \times \Theta(1-\sqrt{r}-\sqrt{\rho}), \\
\mathcal{J}(0,1,1,1,0)= & \left(\frac{4 \pi \bar{\mu}^{2}}{M^{2}}\right)^{2 \epsilon} \frac{M^{2}}{256 \pi^{3}} \frac{\Gamma(-1+\epsilon) \Gamma(1-\epsilon)}{\Gamma(2-2 \epsilon)} \\
& \times\left(x_{+}-x_{-}\right)^{1-2 \epsilon} \Theta(1-\sqrt{r}-\sqrt{\rho}) .
\end{aligned}
$$

Note that $\mathcal{J}(0,1,0,1,1)$ does not contribute to the massless case, since $x_{-} x_{+}=\rho=0$.

The Feynman graph representing the master integral $\mathcal{J}(0,1,1,1,1)$, which is a two-loop four-massive propagator integral with three different masses, is shown in Fig. 4(c). The only way to get a nonzero imaginary part is by cutting the $m$ and $\chi$ propagators. Cutting more lines results in a vanishing contribution by the same phase-space argument discussed above. Since only two propagators are cut, the computation is drastically simplified and the integral can be evaluated analytically in its full $\epsilon$ dependence.

For the explicit computation we can first integrate the loop without cut while taking the "external" $\chi$-propagator momentum to be on-shell $q_{2}^{2}=\chi^{2}$, since we eventually will cut this line. The corresponding integral can be expressed in terms of hypergeometric functions of the kind ${ }_{2} F_{1}$. Finally, we can take the imaginary part of the remaining loop integral by using Eq. (A1). The result reads

$$
\begin{aligned}
\mathcal{J}(0,1,1,1,1)= & \left(\frac{4 \pi \bar{\mu}^{2}}{M^{2}}\right)^{2 \epsilon} \frac{1}{128 \pi^{3}} \frac{\Gamma(1-\epsilon) \Gamma(\epsilon)}{\Gamma(3-2 \epsilon)} \frac{\left(x_{+}-x_{-}\right)^{1-3 \epsilon}\left(-1+x_{+}\right)^{\epsilon}}{1-x_{+}} \\
& \times\left[x_{+}^{1-\epsilon}{ }_{2} F_{1}\left(1-\epsilon, \epsilon ; 2-\epsilon ; \frac{x_{+}\left(1-x_{-}\right)}{x_{+}-x_{-}}\right)-{ }_{2} F_{1}\left(1-\epsilon, \epsilon ; 2-\epsilon ; \frac{1-x_{-}}{x_{+}-x_{-}}\right)\right] \Theta(1-\sqrt{r}-\sqrt{\rho}) .
\end{aligned}
$$

Finally, the Feynman graphs representing the master integrals $\mathcal{J}(1,1,0,0,1), \mathcal{J}(1,2,0,0,1)$, and $\mathcal{J}(2,1,0,0,1)$ are shown in Figs. 4(d)-4(f), respectively. These are two-loop integrals of the sunset type with one massless line and two massive lines of different masses, $\chi$ and $m$. They differ by the powers of the denominators. The master integrals $\mathcal{J}(1,2,0,0,1)$ and $\mathcal{J}(2,1,0,0,1)$ can be related to derivatives of $\mathcal{J}(1,1,0,0,1)$ with respect to the masses 


$$
\begin{gathered}
\mathcal{J}(1,2,0,0,1)=\frac{d}{d \chi^{2}} \mathcal{J}(1,1,0,0,1), \\
\mathcal{J}(2,1,0,0,1)=\frac{1}{M^{4} \epsilon\left(r^{2}+(-1+\rho)^{2}-2 r(1+\rho)\right)}\left[2(-1+\epsilon)^{2} \mathcal{J}(0,1,0,0,1)\right. \\
\\
-M^{2}(-1+2 \epsilon)((-2+3 \epsilon)(-1+r+\rho) \mathcal{J}(1,1,0,0,1) \\
\left.\left.+2 M^{2}\left((-1+\rho) \rho \frac{d}{d m^{2}} \mathcal{J}(1,1,0,0,1)+(-1+r) r \frac{d}{d \chi^{2}} \mathcal{J}(1,1,0,0,1)\right)\right)\right],
\end{gathered}
$$

where Eq. (24) has been obtained by writing $\mathcal{J}(1,1,0,0,2)$ as a combination of the master integrals in Eq. (20) by using LITERED and solving for $\mathcal{J}(2,1,0,0,1)$. Alternatively, in terms of $x_{ \pm}$, the relations to $\mathcal{J}(1,1,0,0,1)$ read

$$
\begin{aligned}
& \mathcal{J}(1,2,0,0,1)=\frac{1}{M^{2}} \frac{1}{x_{+}-x_{-}}\left(x_{-} \frac{\partial}{\partial x_{-}}-x_{+} \frac{\partial}{\partial x_{+}}\right) \mathcal{J}(1,1,0,0,1), \\
& \mathcal{J}(2,1,0,0,1)= \frac{2(1-2 \epsilon)}{M^{2} \epsilon\left(x_{+}-x_{-}\right)^{2}}\left[\frac{1}{2}(2-3 \epsilon)\left(x_{+}+x_{-}-2 x_{+} x_{-}\right) \mathcal{J}(1,1,0,0,1)\right. \\
&+\frac{x_{+}\left(1-x_{+}\right)}{x_{+}-x_{-}}\left(\left(1-x_{+} x_{-}\right) x_{-}+\left(x_{+}+x_{-}-x_{+} x_{-}\right)\left(1-x_{-}\right)\right) \frac{\partial}{\partial x_{+}} \mathcal{J}(1,1,0,0,1) \\
&\left.-\frac{x_{-}\left(1-x_{-}\right)}{x_{+}-x_{-}}\left(\left(1-x_{+} x_{-}\right) x_{+}+\left(x_{+}+x_{-}-x_{+} x_{-}\right)\left(1-x_{+}\right)\right) \frac{\partial}{\partial x_{-}} \mathcal{J}(1,1,0,0,1)\right] .
\end{aligned}
$$

Therefore, the main problem is the computation of $\mathcal{J}(1,1,0,0,1)$. This integral is the most complicated one, since it requires cutting one massless line and two massive lines of different masses. In this particular case, making use of $x_{ \pm}$instead of $r$ and $\rho$ makes a crucial difference, allowing us to compute this contribution also analytically, however, only in an $\epsilon$-expansion.

For the computation we first introduce Feynman parametrization in the massive loop and integrate over the loop momenta. Next we make use of the generalized spectral function with one massive line [30,31]

$$
\begin{aligned}
\operatorname{Im} \frac{1}{i} \int \frac{d^{D} k}{\left(m_{0}^{2}-k^{2}\right)^{a}\left(-(p-k)^{2}\right)^{b}}= & \frac{\pi^{1+D / 2} \Gamma(D / 2-b)}{\Gamma(a) \Gamma(b) \Gamma(D-a-2 b+1)}\left(1-\frac{m_{0}^{2}}{M^{2}}\right)^{D-a-2 b} M^{D-2 a-2 b} \\
& \times{ }_{2} F_{1}\left(D / 2-b, 1-b ; D-a-2 b+1 ; 1-\frac{m_{0}^{2}}{M^{2}}\right)
\end{aligned}
$$

to compute the imaginary part, where in our case

$$
m_{0}^{2}=\frac{m^{2}}{1-x}+\frac{\chi^{2}}{x}
$$

is a function of $m, \chi$, and a single Feynman parameter $x$. The branch cut appears always that $M^{2}>m_{0}^{2}(m, \chi, x)$, which constrains the integration limits of the Feynman parameter integral from $x_{-}$to $x_{+}$, instead of from zero to one. Finally, we end up with an integral of the form

$$
\begin{aligned}
& \mathcal{J}(1,1,0,0,1) \\
& \propto \int_{x_{-}}^{x_{+}} d x(x(1-x))^{-2+2 \epsilon}\left(-\left(x-x_{+}\right)\left(x-x_{-}\right)\right)^{2-3 \epsilon},
\end{aligned}
$$

which cannot be expressed in terms of the usual ${ }_{2} F_{1}$-hypergeometric functions. It requires further study if it can be expressed in terms of generalized ${ }_{p} F_{q}$-hypergeometric functions or if it requires the introduction of the class of functions called elliptic polylogarithms, which are known to appear in two-loop sunset diagrams with three massive lines [32].

However, the integral can be computed as an $\epsilon$ expansion to the necessary order, the reason is related to the fact that one of the lines is massless. In general, we need the master integrals expanded to $\mathcal{O}(\epsilon)$, because the coefficients in front of the master integrals can be proportional to $1 / \epsilon$.

We first note that the integral is finite. The $\mathcal{O}\left(\epsilon^{0}\right)$ term is trivial since it is the integral of a rational function and can be directly computed. The $\mathcal{O}(\epsilon)$ term is an integral of the form $R(x) \ln (P(x))$, where $R(x)$ is a rational function and $P(x)$ a polynomial, and thus the integral can be expressed in terms of dilogarithms [33]. The resulting expression reads 


$$
\begin{aligned}
& \mathcal{J}(1,1,0,0,1)=\frac{M^{2}}{512 \pi^{3}}\left\{[ 1 + \frac { \epsilon } { 2 } ( 9 + 8 \operatorname { l n } ( \frac { \mu } { M } ) ) ] \left[\left(x_{+}-x_{-}\right)\left(2-x_{+}-x_{-}+2 x_{+} x_{-}\right)\right.\right. \\
& \left.-2 x_{-} x_{+}\left(x_{+}+x_{-}-x_{+} x_{-}\right) \ln \left(\frac{x_{+}}{x_{-}}\right)-2\left(1-x_{+}\right)\left(1-x_{-}\right)\left(1-x_{-} x_{+}\right) \ln \left(\frac{1-x_{-}}{1-x_{+}}\right)\right] \\
& +\epsilon\left[2\left(x_{+}-x_{-}\right)\left(2-x_{-}-x_{+}+2 x_{-} x_{+}\right)\left(1-3 \ln \left(x_{+}-x_{-}\right)\right)\right. \\
& +\left(1-x_{+}\right)\left(x_{+} x_{-}\left(1-4 x_{-}\right)-4+x_{+}+x_{-}+x_{-}^{2}\right) \ln \left(1-x_{+}\right) \\
& -\left(1-x_{-}\right)\left(x_{+} x_{-}\left(1-4 x_{+}\right)-4+x_{+}+x_{-}+x_{+}^{2}\right) \ln \left(1-x_{-}\right) \\
& -x_{-}\left(x_{+} x_{-}\left(7-4 x_{+}\right)+4-2 x_{-}-4 x_{+}+3 x_{+}^{2}\right) \ln \left(x_{-}\right) \\
& +x_{+}\left(x_{+} x_{-}\left(7-4 x_{-}\right)+4-2 x_{+}-4 x_{-}+3 x_{-}^{2}\right) \ln \left(x_{+}\right) \\
& +4\left(-2 x_{+} x_{-}\left(x_{-}+x_{+}-x_{+} x_{-}\right)+x_{-}+x_{+}-1\right)\left(\mathrm{Li}_{2}\left(x_{-}\right)-\mathrm{Li}_{2}\left(x_{+}\right)\right) \\
& -2\left(1-x_{-}\right)\left(1-x_{+}\right)\left(1-x_{+} x_{-}\right)\left(3 \operatorname{Li}_{2}\left(\frac{x_{-}-x_{+}}{x_{-}-1}\right)-3 \operatorname{Li}_{2}\left(\frac{x_{+}-x_{-}}{x_{+}-1}\right)+\ln ^{2}\left(1-x_{-}\right)\right. \\
& \left.-\ln ^{2}\left(1-x_{+}\right)+2 \ln \left(1-x_{-}\right) \ln \left(x_{-}\right)-2 \ln \left(1-x_{+}\right) \ln \left(x_{+}\right)+6 \ln \left(\frac{1-x_{+}}{1-x_{-}}\right) \ln \left(x_{+}-x_{-}\right)\right) \\
& -2 x_{+} x_{-}\left(x_{+}+x_{-}-x_{+} x_{-}\right)\left(3 \operatorname{Li}_{2}\left(1-\frac{x_{-}}{x_{+}}\right)-3 \operatorname{Li}_{2}\left(1-\frac{x_{+}}{x_{-}}\right)-\ln ^{2}\left(x_{-}\right)+\ln ^{2}\left(x_{+}\right)\right. \\
& \left.\left.\left.+6 \ln \left(\frac{x_{-}}{x_{+}}\right) \ln \left(x_{+}-x_{-}\right)\right)\right]+\mathcal{O}\left(\epsilon^{2}\right)\right\} \Theta(1-\sqrt{r}-\sqrt{\rho}) \text {. }
\end{aligned}
$$

Once $\mathcal{J}(1,1,0,0,1)$ is known we can compute $\mathcal{J}(1,2,0,0,1)$ and $\mathcal{J}(2,1,0,0,1)$ by using Eqs. (26) and (27). Explicitly, they read

$$
\begin{aligned}
\mathcal{J}(1,2,0,0,1)= & \frac{1}{256 \pi^{3}}\left\{\left[1+4 \epsilon \ln \left(\frac{\mu}{M}\right)\right]\left[x_{+}-x_{-}+x_{+} x_{-} \ln \left(\frac{x_{+}}{x_{-}}\right)+\left(1-x_{+} x_{-}\right) \ln \left(\frac{1-x_{+}}{1-x_{-}}\right)\right]\right. \\
& +\epsilon\left[\left(x_{+}-x_{-}\right)\left(5-6 \ln \left(x_{+}-x_{-}\right)\right)-\left(1+x_{-}\right)\left(1-x_{+}\right) \ln \left(1-x_{-}\right)+\left(1-x_{-}\right)\left(1+x_{+}\right) \ln \left(1-x_{+}\right)\right. \\
& -x_{-}\left(x_{+}+2\right) \ln \left(x_{-}\right)+x_{+}\left(x_{-}+2\right) \ln \left(x_{+}\right)-2\left(1-2 x_{+} x_{-}\right)\left(\operatorname{Li}_{2}\left(x_{-}\right)-\operatorname{Li}_{2}\left(x_{+}\right)\right) \\
& +x_{+} x_{-}\left(3 \operatorname{Li}_{2}\left(1-\frac{x_{-}}{x_{+}}\right)-3 \operatorname{Li}_{2}\left(1-\frac{x_{+}}{x_{-}}\right)+\ln ^{2}\left(x_{+}\right)-\ln ^{2}\left(x_{-}\right)+6 \ln \left(\frac{x_{-}}{x_{+}}\right) \ln \left(x_{+}-x_{-}\right)\right) \\
& -\left(1-x_{+} x_{-}\right)\left(3 \operatorname{Li}_{2}\left(\frac{x_{-}-x_{+}}{x_{-}-1}\right)-3 \operatorname{Li}_{2}\left(\frac{x_{+}-x_{-}}{x_{+}-1}\right)+6 \ln \left(\frac{1-x_{+}}{1-x_{-}}\right) \ln \left(x_{+}-x_{-}\right)+\ln ^{2}\left(1-x_{-}\right)-\ln ^{2}\left(1-x_{+}\right)\right. \\
& \left.\left.\left.+2 \ln \left(1-x_{-}\right) \ln \left(x_{-}\right)-2 \ln \left(1-x_{+}\right) \ln \left(x_{+}\right)\right)\right]+\mathcal{O}\left(\epsilon^{2}\right)\right\} \Theta(1-\sqrt{r}-\sqrt{\rho}), \\
& \mathcal{J}(2,1,0,0,1)=\frac{1}{256 \pi^{3}}\left\{\left(x_{+}-x_{-}\right)\left[\frac{1}{\epsilon}+4 \ln \left(\frac{\mu}{M}\right)-6 \ln \left(x_{+}-x_{-}\right)+3\right]\right. \\
& +\left(1-x_{-}\right)\left(1+x_{+}\right) \ln \left(1-x_{-}\right)-\left(1-x_{+}\right)\left(1+x_{-}\right) \ln \left(1-x_{+}\right) \\
& \left.+x_{-}\left(x_{+}-2\right) \ln \left(x_{-}\right)-x_{+}\left(x_{-}-2\right) \ln \left(x_{+}\right)+\mathcal{O}(\epsilon)\right\} \Theta(1-\sqrt{r}-\sqrt{\rho}) .
\end{aligned}
$$

Note that $\mathcal{J}(2,1,0,0,1) \sim(1 / \epsilon) \mathcal{J}(1,1,0,0,1)$. Therefore, in order to compute $\mathcal{J}(2,1,0,0,1)$ to $\mathcal{O}(\epsilon)$ we need $\mathcal{J}(1,1,0,0,1)$ to $\mathcal{O}\left(\epsilon^{2}\right)$, whose computation can be rather cumbersome but possible. Due to the length of the resulting expression we do not present it explicitly but we provide it upon request. 
However, for the particular applications we will discuss in Sec. IV the coefficient in front of $\mathcal{J}(2,1,0,0,1)$ coming from the integration by parts reduction is $\mathcal{O}\left(\epsilon^{0}\right)$, so the integral is only needed to $\mathcal{O}\left(\epsilon^{0}\right)$, and thus knowing $\mathcal{J}(1,1,0,0,1)$ to $\mathcal{O}(\epsilon)$ is actually enough.

Note that some of the master integrals are divergent as $\epsilon \rightarrow 0$, so in principle there can even be $1 / \epsilon^{2}$ terms. However, in the expressions for physical quantities such as the differential widths the master integrals must combine in such a way that the $1 / \epsilon^{2}$ term cancels. This is to be expected since after taking cut we face one-loop integrals, so $1 / \epsilon^{2}$ terms should never appear. A different way of seeing this is that at $\mathcal{O}\left(\alpha_{s}\right)$ poles must cancel with the oneloop renormalization factors of the fields and masses which, to this order, contain a simple pole in $\epsilon$ at most. The explicit cancellation of $1 / \epsilon^{2}$ terms in the applications discussed in Sec. IV is thus a check of our calculation.

Let us mention that there is a peculiarity related to the $1 / \epsilon^{2}$ terms. In a one-loop calculation it is usually enough to take only the finite piece which involves only logarithms. However, since our original integrals contain $1 / \epsilon^{2}$, which is a two-loop feature, the finite piece contains also dilogarithms and factors of $\pi^{2}$. Even though in general the $1 / \epsilon^{2}$ cancels in the width, a combination of dilogarithms and factors of $\pi^{2}$ survive. These surviving terms are a remnant of the extra divergences which appeared in intermediate steps. These extra divergences are related to the fact that the $q$ quark is on-shell. A similar mechanism is at work in the matching of heavy-light currents in soft collinear effective theory and collinear divergences [34].

Finally, we have explicitly checked that our results for the master integrals reproduce the ones in the massless case, which are computed below in Sec. III E.

\section{LO-QCD (Massless channel)}

At LO-QCD the differential decay width for the massless $(\rho=0)$ channel can be written as a combination of oneloop two-propagator integrals with a single mass $\chi$ and onshell external momenta $p^{2}=M^{2}$ of the form

$$
\mathcal{M}\left(n_{1}, n_{2}\right) \equiv \operatorname{Im} \bar{\mu}^{2 \epsilon} \frac{1}{i} \int \frac{d^{D} q}{(2 \pi)^{D}} \frac{1}{\left(q^{2}-\chi^{2}\right)^{n_{1}}\left((p-q)^{2}\right)^{n_{2}}} .
$$

By using LITERED we reduce every integral above as a combination of the two master integrals $\mathcal{M}(1,0)$ and $\mathcal{M}(1,1)$. The master integral $\mathcal{M}(1,0)$ is a closed massive loop and its imaginary part is zero. The master integral $\mathcal{M}(1,1)$ is a one-loop integral with one massive and one massless line, whose associated graph is displayed in Fig. 5(a). It can easily be computed by using the dispersion representation given in Eq. (A1). It reads
$\mathcal{M}(1,1)$

$$
=\left(\frac{4 \pi \bar{\mu}^{2}}{M^{2}}\right)^{\epsilon} \frac{1}{16 \pi} \frac{\Gamma(1-\epsilon)}{\Gamma(2-2 \epsilon)}(1-r)^{1-2 \epsilon} \Theta(1-r) .
$$

\section{E. NLO-QCD (Massless channel)}

At NLO-QCD the differential decay width for the massless $(\rho=0)$ channel can be written as a combination of two-loop five-propagator integrals with two different masses $\chi$ and $M$ and on-shell external momenta $p^{2}=M^{2}$ of the form

$$
\begin{aligned}
& \mathcal{J}\left(n_{1}, n_{2}, n_{3}, n_{4}, n_{5}\right) \\
& \quad \equiv \operatorname{Im} \bar{\mu}^{4 \epsilon} \int \frac{d^{D} q_{1}}{(2 \pi)^{D}} \frac{d^{D} q_{2}}{(2 \pi)^{D}} \frac{1}{D_{1}^{n_{1}} D_{2}^{n_{2}} D_{3}^{n_{3}} D_{4}^{n_{4}} D_{5}^{n_{5}}},
\end{aligned}
$$

where

$D_{1}=q_{1}^{2}, \quad D_{2}=q_{2}^{2}-\chi^{2}, \quad D_{3}=\left(p-q_{1}\right)^{2}-M^{2}$,

$D_{4}=\left(p-q_{2}\right)^{2}, \quad D_{5}=\left(p-q_{1}-q_{2}\right)^{2}$.

By using LITERED we reduce every integral above as a combination of the following seven master integrals

$$
\begin{array}{cc}
\mathcal{J}(0,1,1,1,0), & \mathcal{J}(0,0,1,1,1), \\
\mathcal{J}(0,1,1,1,1), & \mathcal{J}(0,1,1,0,0), \\
\mathcal{J}(1,1,0,0,1), & \mathcal{J}(0,1,1,0,1), \\
\mathcal{J}(2,1,0,0,1) .
\end{array}
$$

The master integrals in the right column are either closed loops or two-loop sunset type diagrams which-after taking the cut-vanish by the same arguments as for the massive case. They are either massive tadpoles (which are real) or there is no phase space. Again, we have checked this statement by explicit calculation as well.

The master integral $\mathcal{J}(0,1,1,1,0)$ is factorizable in the product of the cut one-loop sunset integral with one massive line $\chi$, computed in Sec. III D, times a closed massive loop with mass $M$. Any other cut is not possible. The corresponding topology is shown in Fig. 5(b). The result reads

$$
\begin{aligned}
\mathcal{J}(0,1,1,1,0)= & \left(\frac{4 \pi \bar{\mu}^{2}}{M^{2}}\right)^{2 \epsilon} \frac{M^{2}}{256 \pi^{3}} \frac{\Gamma(-1+\epsilon) \Gamma(1-\epsilon)}{\Gamma(2-2 \epsilon)} \\
& \times(1-r)^{1-2 \epsilon} \Theta(1-r) .
\end{aligned}
$$

The Feynman graph representing the master integral $\mathcal{J}(0,1,1,1,1)$, which is a two-loop two-massless and two-massive propagator integral with two different masses, is shown in Fig. 5(c). For the computation we can proceed 

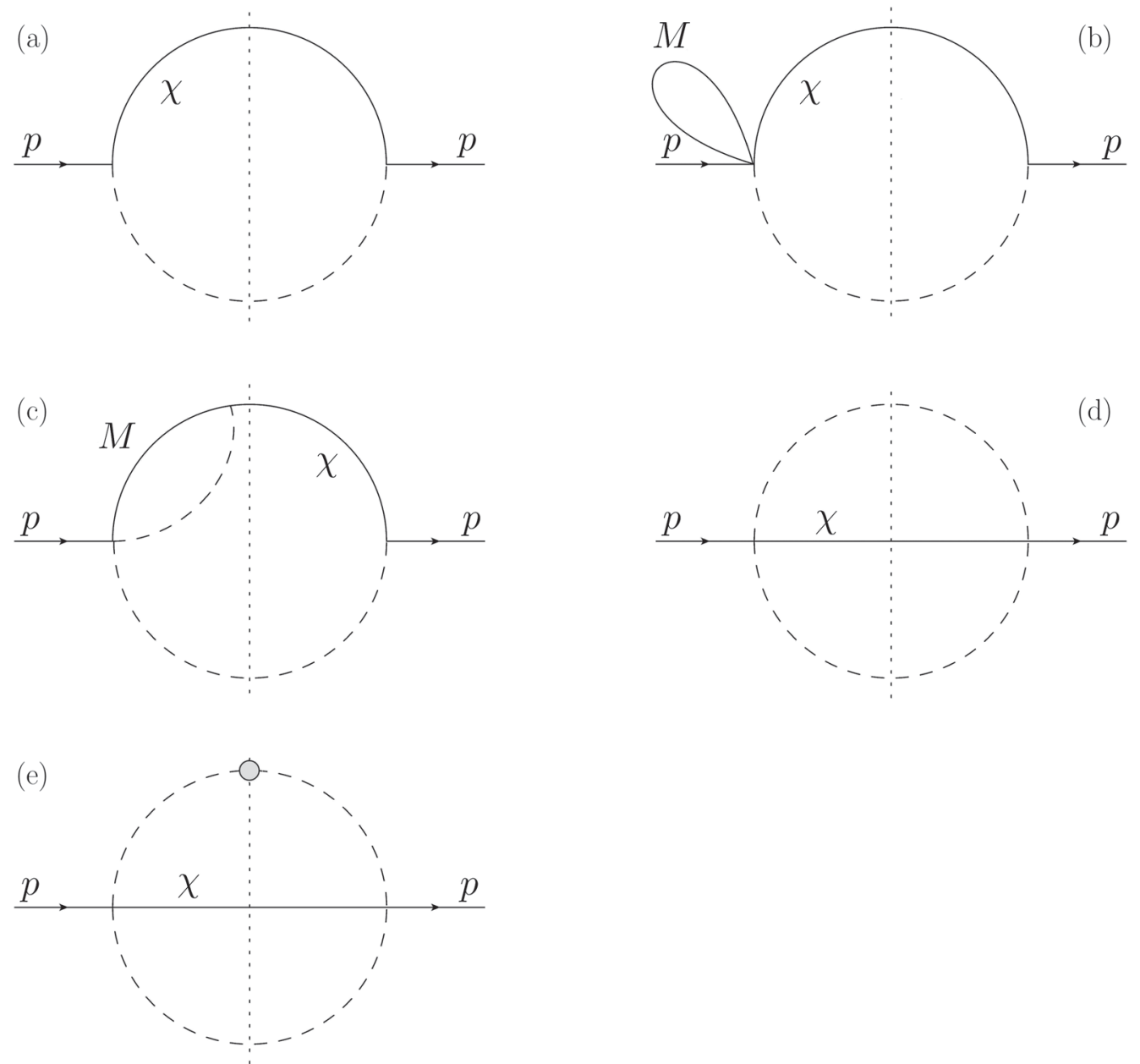

FIG. 5. Master integral topologies stemming from the massless decay channel contributing to the differential decay width up to NLOQCD. Continuous and dashed lines stand for massive and massless propagators, respectively. The gray dot stands for iteration of the corresponding propagator, and the vertical dotted line represents a cut.

exactly as in the completely massive case. The only way to get a nonzero imaginary part is by cutting the massless and $\chi$ propagators. Otherwise, there is not enough energy in the initial state to produce the final state particles (one particle of mass $M$ and two massless quarks). Again, the cut involves only two propagators. Since the loop involving the particle of mass $M$ and one massless particle is not cut, we can first integrate it while taking the "external" $\chi$ propagator momentum to be on-shell due to the cut on it. Finally, we can take the imaginary part of the remaining loop integral by using Eq. (A1). The result reads

$$
\begin{aligned}
\mathcal{J}(0,1,1,1,1)= & -\left(\frac{4 \pi \bar{\mu}^{2}}{M^{2}}\right)^{2 \epsilon} \frac{1}{128 \pi^{3}} \frac{\Gamma(1-\epsilon) \Gamma(\epsilon)}{\Gamma(3-2 \epsilon)} \\
& \times(1-r)^{1-2 \epsilon}{ }_{2} F_{1}(1, \epsilon ; 2-\epsilon ; r) \\
& \times \Theta(1-r) .
\end{aligned}
$$

Finally, the Feynman graphs representing the master integrals $\mathcal{J}(1,1,0,0,1)$ and $\mathcal{J}(2,1,0,0,1)$ are shown in
Figs. 5(d) and 5(e). These are two-loop integrals of the sunset type with two massless lines and one massive line of mass $\chi$. They differ by the powers of the denominators. The master integral $\mathcal{J}(2,1,0,0,1)$ can be related to $\mathcal{J}(1,1,0,0,1)$ and derivatives of it with respect to the mass $\chi$. Explicitly

$$
\begin{aligned}
\mathcal{J}(2,1,0,0,1) \\
=\frac{(-3+D)}{(-4+D)\left(M^{2}-\chi^{2}\right)}[(8-3 D) \mathcal{J}(1,1,0,0,1) \\
\left.\quad+4 \chi^{2} \frac{d}{d \chi^{2}} \mathcal{J}(1,1,0,0,1)\right] .
\end{aligned}
$$

To obtain the relation above we reduce the integral $\mathcal{J}(1,2,0,0,1)=\frac{d}{d \chi^{2}} \mathcal{J}(1,1,0,0,1)$ using LITERED and invert the relation to find $\mathcal{J}(2,1,0,0,1)$ in terms of $\mathcal{J}(1,2,0,0,1)$ and $\mathcal{J}(1,1,0,0,1)$. 
Therefore, the problem is reduced to the computation of $\mathcal{J}(1,1,0,0,1)$. It requires taking a cut across two massless lines and one massive line. For the computation we can proceed analogously to the massive case. Since there is only one massive line, the integration can be performed to all orders in $\epsilon$, unlike in the massive case. The result reads

$$
\mathcal{J}(1,1,0,0,1)=\left(\frac{4 \pi \bar{\mu}^{2}}{M^{2}}\right)^{2 \epsilon} \frac{M^{2}}{256 \pi^{3}} \frac{\Gamma^{2}(1-\epsilon)}{\Gamma(4-4 \epsilon)}(1-r)^{3-4 \epsilon}{ }_{2} F_{1}(2-2 \epsilon, 1-\epsilon ; 4-4 \epsilon ; 1-r) \Theta(1-r) .
$$

Once $\mathcal{J}(1,1,0,0,1)$ is known, we can compute $\mathcal{J}(2,1,0,0,1)$ by using Eq. (44). It reads

$$
\begin{aligned}
\mathcal{J}(2,1,0,0,1)= & \left(\frac{4 \pi \bar{\mu}^{2}}{M^{2}}\right)^{2 \epsilon} \frac{1}{256 \pi^{3}} \frac{\Gamma(2 \epsilon) \Gamma(2-\epsilon) \Gamma(-1+\epsilon) \Gamma(1-\epsilon)}{\Gamma(1+\epsilon) \Gamma(4-4 \epsilon) \Gamma(-1+2 \epsilon)} \\
& \times(1-r)^{1-4 \epsilon}\left[(2-3 \epsilon+r(4-5 \epsilon))_{2} F_{1}(2-2 \epsilon, 1-\epsilon ; 4-4 \epsilon ; 1-r)\right. \\
& \left.+(1-\epsilon) r(1-r)_{2} F_{1}(3-2 \epsilon, 2-\epsilon ; 5-4 \epsilon ; 1-r)\right] \Theta(1-r) .
\end{aligned}
$$

Unlike in the massive channel case, we are able to get all master integrals in their full $\epsilon$ dependence.

\section{APPLICATIONS}

As a sample application, we compute the differential and total $B$-hadron $\left(Q=b, M=m_{b}\right)$ semileptonic decay width to leading order in the $1 / m_{b}$ expansion. There are two decay channels that contribute. On the one hand, there is a Cabibbo favored transition $b \rightarrow c \ell \bar{\nu}_{\ell}$, where we identify $q=c$ and $m=m_{c}$. On the other hand, there is a Cabibbo suppressed transition $b \rightarrow u \ell \bar{\nu}_{\ell}$, where we identify $q=u$ and $m=0$. They correspond to the massive and massless cases, respectively, which have been discussed in Secs. II and III. In both channels $n=2$ with $p_{1}=\ell$ and $p_{2}=\bar{\nu}_{\ell}$ being massless leptons, $m_{1}=m_{2}=0$. Again, we use the massless case as a check of the computation for the massive case in the limit $\rho \rightarrow 0$.

The effective electroweak Lagrangian describing the flavor changing transition $b \rightarrow q \ell \bar{\nu}_{\ell}$ reads [35]

$$
\begin{aligned}
\mathcal{L}_{\mathrm{EW}, \mathrm{eff}}= & -\frac{4 G_{F}}{\sqrt{2}} \sum_{q=c, u}\left(V_{q b}\left(\bar{b} \Gamma_{\mu} q\right)\left(\bar{\nu}_{\ell} \Gamma^{\mu} \ell\right)\right. \\
& \left.+V_{q b}^{\dagger}\left(\bar{q} \Gamma_{\mu} b\right)\left(\bar{\ell} \Gamma^{\mu} \nu_{\ell}\right)\right)
\end{aligned}
$$

where $G_{F}$ is the Fermi constant, $V_{q Q}$ is the corresponding CKM matrix element describing weak mixing of quark generations, and $\Gamma^{\mu}=\gamma^{\mu} P_{L}$ with $P_{L}=\frac{1}{2}\left(1-\gamma_{5}\right)$ being the left-handed projector. In the cases under study $\Gamma^{0}=$ $G_{F}^{2} m_{b}^{5}\left|V_{q b}\right|^{2} /\left(192 \pi^{3}\right)$.

Before going to the technical details we need to discuss how renormalization will be performed. For light-quark masses and the strong coupling $\alpha_{s}(\mu)$ we adopt the $\overline{\mathrm{MS}}$ renormalization scheme [36]. The bottom and charm quarks will be renormalized on-shell. In practice, that is $b_{B}=\left(Z_{2}^{\mathrm{OS}}\right)^{1 / 2} b$ and $m_{c, B}=Z_{m_{c}}^{\mathrm{OS}} m_{c}^{\text {pole }}$, where quantities with omitted indices stand for renormalized ones, and

$$
Z_{m_{q}}^{\mathrm{OS}}=1-C_{F} \frac{\alpha_{s}(\mu)}{4 \pi}\left(\frac{3}{\epsilon}+6 \ln \left(\frac{\mu}{m_{q}}\right)+4\right),
$$

with $Z_{2}^{\mathrm{OS}}=Z_{m_{b}}^{\mathrm{OS}}$ to this order. Also $g_{s, B}^{2}=4 \pi Z_{g}^{2} \alpha_{s}(\mu) \bar{\mu}^{2 \epsilon}$. For the precision of the calculation the renormalization factor of the strong coupling is only needed at tree level $\left(Z_{g}=1\right)$. The quantity $C_{F}=4 / 3$ is a color factor.

Note that for the charm quark it is still not so clear if it is better to perform the renormalization in the on-shell or the $\overline{\mathrm{MS}}$ scheme. We have chosen the former because results become slightly more compact in that scheme. However, since $m_{c}$ is better known in the $\overline{\mathrm{MS}}$ scheme, it might be useful to have the results with $m_{c}$ in that scheme. This can easily be achieved by using the relation between the $\overline{\mathrm{MS}}$ and pole masses at one-loop order,

$$
m_{c}^{\text {pole }}=m_{c}^{\mathrm{MS}}(\mu)\left(1+C_{F} \frac{\alpha_{s}}{4 \pi}\left(6 \ln \left(\frac{\mu}{m_{c}}\right)+4\right)\right) .
$$

Note that LO-QCD diagrams not only contribute to this order, but also to NLO-QCD after replacing the bare bottom field and charm quark mass by their renormalized counterparts.

The Feynman diagrams that contribute to the total semileptonic width at LO-QCD and NLO-QCD are the two-loop and three-loop diagrams shown in Fig. 6. Following Eq. (3), we use the dispersion representation of the one-loop sunset topology given in Eqs. (A1) and (A2) to write the lepton-neutrino loop represented in Fig. 7 as an integral differential in the lepton pair invariant mass square $\chi^{2}$. That is, if both leptons are massless

$$
\begin{aligned}
& \int \frac{d^{D} k}{(2 \pi)^{D}} \frac{-\operatorname{Tr}\left(\Gamma^{\sigma} i\left(\not k+\not q_{2}\right) \Gamma^{\rho} i \not k\right)}{k^{2}\left(k+q_{2}\right)^{2}} \\
& \quad=i \int_{0}^{\infty} d\left(\chi^{2}\right) \frac{\rho_{s}\left(\chi^{2}\right)}{\chi^{2}-q_{2}^{2}-i \eta}\left(q_{2}^{2} g^{\rho \sigma}-q_{2}^{\rho} q_{2}^{\sigma}\right),
\end{aligned}
$$



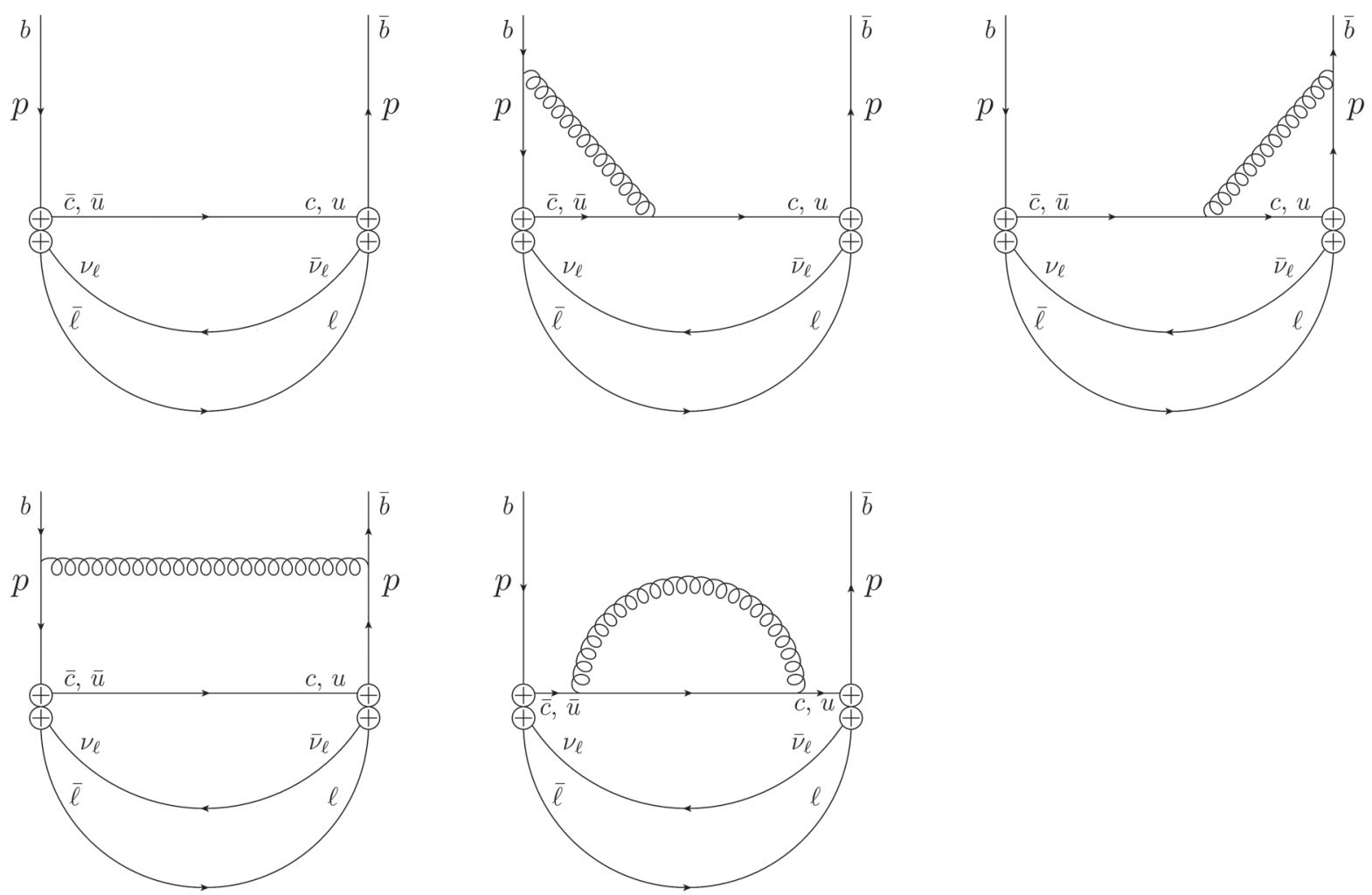

FIG. 6. LO-QCD and NLO-QCD diagrams contributing to the total semileptonic decay width.
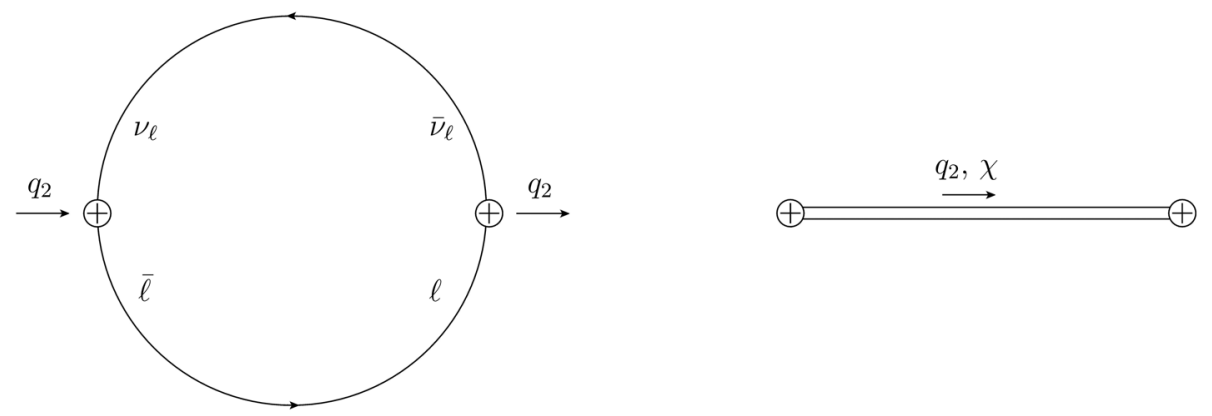

FIG. 7. Graphs representing the leptonic loop appearing in the forward scattering matrix element of the $B$-hadron in usual (left) and dispersion (right) representation.

where the spectral density $\rho_{s}$ reads

$$
\rho_{s}=\frac{D-2}{D-1} \rho_{1 \mathrm{LS}}\left(\chi^{2}, 0,0\right)=\frac{2}{3} \frac{1}{16 \pi^{2}}+\mathcal{O}(\epsilon) .
$$

Since renormalization can be performed at the differential level, the integrand is finite, and it is enough to keep the $\mathcal{O}\left(\epsilon^{0}\right)$ term in $\rho_{s}$. A nice feature of the semileptonic width is that the purely leptonic part is not affected by QCD corrections, which keeps the spectral density very simple. Note that the "effective massive propagator" of mass $\chi$ is transverse due to the leptons are massless. After writing the leptonic loop in this form, we can compute the width differential in the dilepton pair invariant mass square by leaving the integral over $\chi^{2}$ undone.

The Feynman diagrams contributing to the semileptonic differential width are shown in Fig. 8. The corresponding amplitude is reduced to a combination of the master integrals computed in Sec. III by using LITERED. In the $b \rightarrow c \ell \bar{\nu}_{\ell}$ channel, the amplitude at LO-QCD and NLOQCD is written as a combination of the master integrals given in Secs. III B and III C, respectively. In the $b \rightarrow u \ell \bar{\nu}_{\ell}$ channel, the amplitude at LO-QCD and NLO-QCD is written as a combination of the master integrals given in Secs. III D and IIIE, respectively. Whereas the master integrals are universal objects, the particular combination 

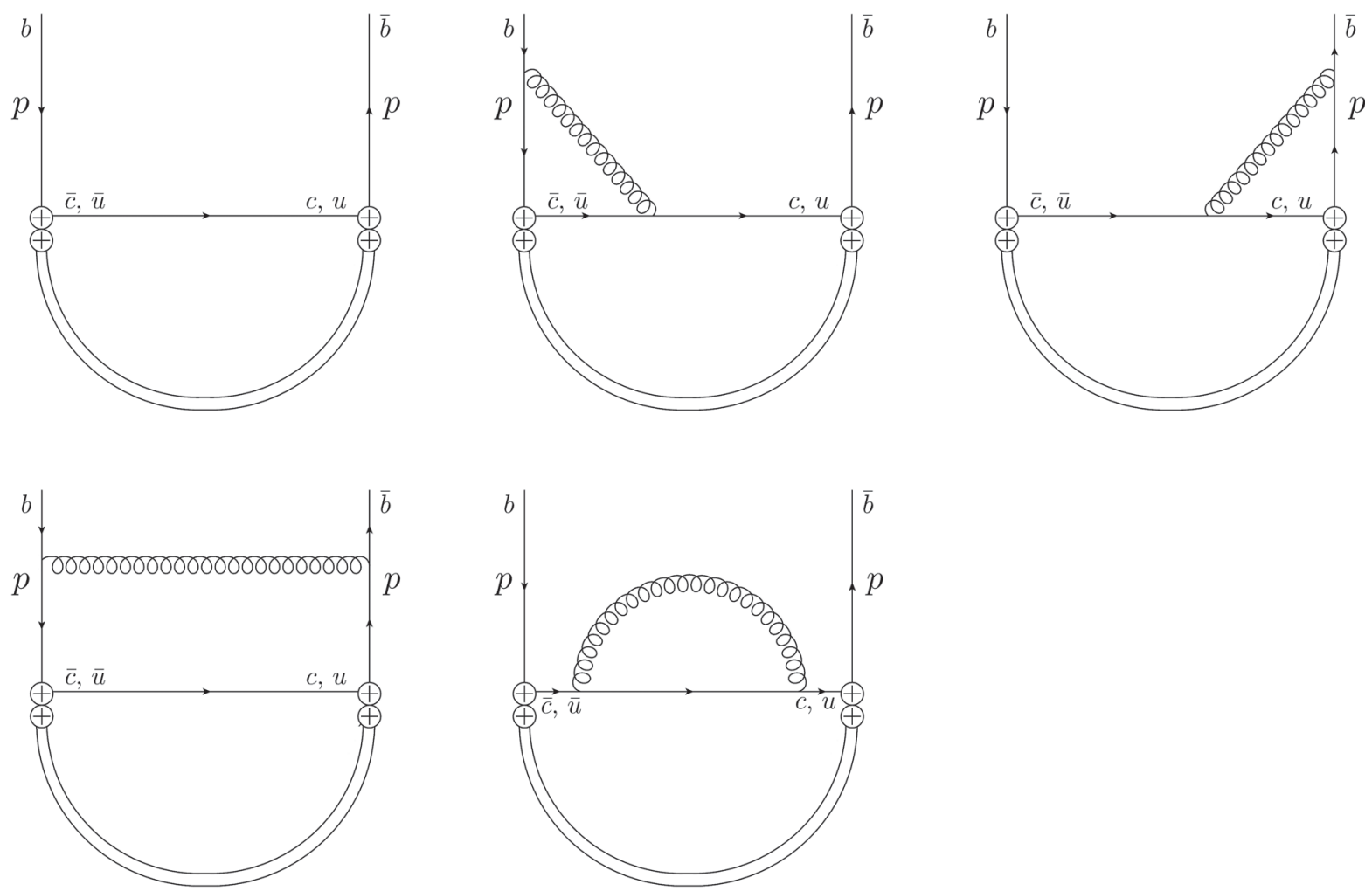

FIG. 8. LO-QCD and NLO-QCD diagrams contributing to the differential semileptonic decay width.

that gives the semileptonic width depends on the structure of the interaction.

The topology of the graphs is such that the NLO contribution can be proportional only to the color factor $C_{F}$.

The computation is done in a general covariant gauge with gauge fixing parameter $a$. The coefficients of the differential width are independent of the gauge fixing parameter, which is a strong check of the calculation.

Whereas the computation of $\alpha_{s}$ corrections to the semileptonic decay width stemming from the massive channel requires computing three loop integrals with one scale $(\rho)$, the computation of the differential width requires one to evaluate two-loop integrals with two scales $(r, \rho)$. That makes the analytical structure of the differential width more involved. Integrating over the additional scale $r$ one obtains the total width. Clearly, the additional integration makes the analytical structure of the total width simpler compared to its differential counterpart, since it is a function of one less parameter.

\section{RESULTS}

In this section we summarize the analytical results for the HQE coefficients of the differential and total (integrated) semileptonic decay width stemming from both the $b \rightarrow$ $c \ell \bar{\nu}_{\ell}$ and the $b \rightarrow u \ell \bar{\nu}_{\ell}$ decay channels with massless leptons. Since we neglect the lepton masses, all $\eta_{i}$ vanish, so the differential rate is a function of $\rho$ and $r$, where $r$ is the invariant mass of the leptons. Integrating over $r$ yields the total rate, which then depends on $\rho$ only.

For presentation, we split the LO and NLO contributions. We define the coefficients of the differential width to be

$$
\mathcal{C}_{0}(\rho, r)=\mathcal{C}_{0}^{\mathrm{LO}}(\rho, r)+C_{F} \frac{\alpha_{s}}{\pi} \mathcal{C}_{0}^{\mathrm{NLO}}(\rho, r)
$$

and the coefficients of the total (integrated) width to be

$$
C_{0}(\rho)=C_{0}^{\mathrm{LO}}(\rho)+C_{F} \frac{\alpha_{s}}{\pi} C_{0}^{\mathrm{NLO}}(\rho) .
$$

\section{A. Differential decay width}

For the $b \rightarrow c \ell \bar{\nu}_{\ell}$ channel the LO and NLO coefficients are most conveniently expressed in the variables $x_{ \pm}$and read

$$
\mathcal{C}_{0}^{\mathrm{LO}}=48 \pi^{2}\left(x_{+}-x_{-}\right)\left(x_{-} x_{+}\left(3 x_{-}+3 x_{+}-8\right)+x_{-}\left(3-2 x_{-}\right)+x_{+}\left(3-2 x_{+}\right)\right) \text {, }
$$




$$
\begin{aligned}
& \mathcal{C}_{0}^{\mathrm{NLO}}=-24 \pi^{2}\left\{\frac{1}{2}\left(x_{-}-x_{+}\right)\left(x_{-} x_{+}\left(8 x_{-} x_{+}+3 x_{-}+3 x_{+}-28\right)+3 x_{-}\left(1-2 x_{-}\right)+3 x_{+}\left(1-2 x_{+}\right)+8\right)\right. \\
& +2\left(x_{-} x_{+}\left(3 x_{-}+3 x_{+}-8\right)+x_{-}\left(3-2 x_{-}\right)+x_{+}\left(3-2 x_{+}\right)\right) \\
& \times\left[( x _ { - } + x _ { + } ) \left(2 \mathrm{Li}_{2}\left(1-\frac{x_{-}}{x_{+}}\right)-2 \mathrm{Li}_{2}\left(1-\frac{x_{+}}{x_{-}}\right)+2 \mathrm{Li}_{2}\left(x_{-}\right)-2 \mathrm{Li}_{2}\left(x_{+}\right)\right.\right. \\
& +2 \ln \left(x_{-}\right) \ln \left(x_{+}-x_{-}\right)-2 \ln \left(x_{+}\right) \ln \left(x_{+}-x_{-}\right)-\ln ^{2}\left(x_{-}\right)+\ln ^{2}\left(x_{+}\right) \\
& \left.\left.+\ln \left(1-x_{-}\right) \ln \left(x_{-}\right)-\ln \left(1-x_{+}\right) \ln \left(x_{+}\right)\right)-4\left(x_{-}-x_{+}\right) \ln \left(x_{+}-x_{-}\right)\right] \\
& +x_{-}\left(x_{-}^{2} x_{+}^{2}\left(4 x_{+}-14\right)+x_{-} x_{+}\left(-14 x_{+}^{2}+34 x_{+}+26 x_{-}-40\right)\right. \\
& \left.+x_{-}\left(15-12 x_{-}\right)+x_{+}\left(5 x_{+}^{2}-4 x_{+}+6\right)\right) \ln \left(x_{-}\right) \\
& -x_{+}\left(x_{-}^{2} x_{+}^{2}\left(4 x_{-}-14\right)+x_{-} x_{+}\left(-14 x_{-}^{2}+34 x_{-}+26 x_{+}-40\right)\right. \\
& \left.+x_{+}\left(15-12 x_{+}\right)+x_{-}\left(5 x_{-}^{2}-4 x_{-}+6\right)\right) \ln \left(x_{+}\right) \\
& -\left(4 x_{-}^{3} x_{+}^{3}-x_{-}^{2} x_{+}^{2}\left(14 x_{-}+14 x_{+}-28\right)+x_{-} x_{+}\left(14 x_{+}^{2}-12 x_{+}+2 x_{-}^{2}+12 x_{-}-28\right)\right. \\
& \left.+x_{-}\left(4 x_{-}^{2}-14 x_{-}+14\right)+x_{+}\left(-4 x_{+}^{2}-2 x_{+}+14\right)-4\right) \ln \left(1-x_{-}\right) \\
& +\left(4 x_{-}^{3} x_{+}^{3}-x_{-}^{2} x_{+}^{2}\left(14 x_{-}+14 x_{+}-28\right)+x_{-} x_{+}\left(14 x_{-}^{2}-12 x_{-}+2 x_{+}^{2}+12 x_{+}-28\right)\right. \\
& \left.\left.+x_{+}\left(4 x_{+}^{2}-14 x_{+}+14\right)+x_{-}\left(-4 x_{-}^{2}-2 x_{-}+14\right)-4\right) \ln \left(1-x_{+}\right)\right\} \text {. }
\end{aligned}
$$

Indeed, the terms involving $\operatorname{Li}_{2}(x)$ and $\ln ^{2}(x)$ can be written more compactly using that

$$
\begin{aligned}
2 \mathrm{Li}_{2} & \left(1-\frac{x_{-}}{x_{+}}\right)-2 \mathrm{Li}_{2}\left(1-\frac{x_{+}}{x_{-}}\right)+2 \mathrm{Li}_{2}\left(x_{-}\right)-2 \mathrm{Li}_{2}\left(x_{+}\right)+2 \ln \left(x_{-}\right) \ln \left(x_{+}-x_{-}\right) \\
& -2 \ln \left(x_{+}\right) \ln \left(x_{+}-x_{-}\right)-\ln ^{2}\left(x_{-}\right)+\ln ^{2}\left(x_{+}\right)+\ln \left(1-x_{-}\right) \ln \left(x_{-}\right)-\ln \left(1-x_{+}\right) \ln \left(x_{+}\right) \\
= & \frac{\pi^{2}}{3}+2 \mathrm{Li}_{2}\left(1-\frac{x_{-}}{x_{+}}\right)-2 \mathrm{Li}_{2}\left(\frac{x_{-}}{x_{+}}\right)+\mathrm{Li}_{2}\left(x_{-}\right)-\mathrm{Li}_{2}\left(1-x_{-}\right)-\mathrm{Li}_{2}\left(x_{+}\right)+\mathrm{Li}_{2}\left(1-x_{+}\right) \\
= & 4 \mathrm{~L}_{\mathrm{R}}\left(1-\frac{x_{-}}{x_{+}}\right)+2 \mathrm{~L}_{\mathrm{R}}\left(x_{-}\right)-2 \mathrm{~L}_{\mathrm{R}}\left(x_{+}\right)
\end{aligned}
$$

where $\mathrm{L}_{\mathrm{R}}(x)$ is Roger's dilogarithm [33]

$$
\mathrm{L}_{\mathrm{R}}(x)=\mathrm{Li}_{2}(x)+\frac{1}{2} \ln (x) \ln (1-x)=\frac{1}{2}\left(\frac{\pi^{2}}{6}+\mathrm{Li}_{2}(x)-\mathrm{Li}_{2}(1-x)\right), \quad 0<x<1 .
$$

The second equality in Eq. (56) displays an explicit $\pi^{2}$ term which highlights the close relationship between $\pi^{2}$ terms and dilogarithms. For example, $\mathrm{Li}_{2}(1)=\pi^{2} / 6$. As we will see throughout this section, we can always hide the explicit $\pi^{2}$ terms by choosing a proper set of dilogarithms to express the results. 
In terms of Roger's dilogarithm the $\mathcal{C}_{0}^{\mathrm{NLO}}$ coefficient reads

$$
\begin{aligned}
\mathcal{C}_{0}^{\mathrm{NLO}}= & -24 \pi^{2}\left\{\frac{1}{2}\left(x_{-}-x_{+}\right)\left(x_{-} x_{+}\left(8 x_{-} x_{+}+3 x_{-}+3 x_{+}-28\right)+3 x_{-}\left(1-2 x_{-}\right)+3 x_{+}\left(1-2 x_{+}\right)+8\right)\right. \\
& +4\left(x_{-} x_{+}\left(3 x_{-}+3 x_{+}-8\right)+x_{-}\left(3-2 x_{-}\right)+x_{+}\left(3-2 x_{+}\right)\right) \\
& \times\left[\left(x_{-}+x_{+}\right)\left(2 \mathrm{~L}_{\mathrm{R}}\left(1-\frac{x_{-}}{x_{+}}\right)+\mathrm{L}_{\mathrm{R}}\left(x_{-}\right)-\mathrm{L}_{\mathrm{R}}\left(x_{+}\right)\right)-2\left(x_{-}-x_{+}\right) \ln \left(x_{+}-x_{-}\right)\right] \\
& +x_{-}\left(x_{-}^{2} x_{+}^{2}\left(4 x_{+}-14\right)+x_{-} x_{+}\left(-14 x_{+}^{2}+34 x_{+}+26 x_{-}-40\right)\right. \\
& \left.+x_{-}\left(15-12 x_{-}\right)+x_{+}\left(5 x_{+}^{2}-4 x_{+}+6\right)\right) \ln \left(x_{-}\right) \\
& -x_{+}\left(x_{-}^{2} x_{+}^{2}\left(4 x_{-}-14\right)+x_{-} x_{+}\left(-14 x_{-}^{2}+34 x_{-}+26 x_{+}-40\right)\right. \\
& \left.+x_{+}\left(15-12 x_{+}\right)+x_{-}\left(5 x_{-}^{2}-4 x_{-}+6\right)\right) \ln \left(x_{+}\right) \\
& -\left(4 x_{-}^{3} x_{+}^{3}-x_{-}^{2} x_{+}^{2}\left(14 x_{-}+14 x_{+}-28\right)+x_{-} x_{+}\left(14 x_{+}^{2}-12 x_{+}+2 x_{-}^{2}+12 x_{-}-28\right)\right. \\
& \left.+x_{-}\left(4 x_{-}^{2}-14 x_{-}+14\right)+x_{+}\left(-4 x_{+}^{2}-2 x_{+}+14\right)-4\right) \ln \left(1-x_{-}\right) \\
& +\left(4 x_{-}^{3} x_{+}^{3}-x_{-}^{2} x_{+}^{2}\left(14 x_{-}+14 x_{+}-28\right)+x_{-} x_{+}\left(14 x_{-}^{2}-12 x_{-}+2 x_{+}^{2}+12 x_{+}-28\right)\right. \\
& \left.\left.+x_{+}\left(4 x_{+}^{2}-14 x_{+}+14\right)+x_{-}\left(-4 x_{-}^{2}-2 x_{-}+14\right)-4\right) \ln \left(1-x_{+}\right)\right\} .
\end{aligned}
$$

Note that, in terms of $x_{ \pm}$, the coefficient of the differential width can be expressed as a function of simple structures which are polynomials or polynomials multiplied by logarithms, square logarithms, and dilogarithms. Alternatively, we can replace the dependence in square logarithms and dilogarithms in favor of Roger's dilogarithms.

It is also interesting to note the remarkable $x_{+} \leftrightarrow x_{-}$ asymmetry. More precisely, we observe that the quantity $\operatorname{Re}\left[\mathcal{C}_{0}\left(x_{+}, x_{-}\right)+\mathcal{C}_{0}\left(x_{-}, x_{+}\right)\right]=0$.

The coefficients for the $b \rightarrow u \ell \bar{\nu}_{\ell}$ channel can be obtained from the expressions for the $b \rightarrow c \ell \bar{\nu}_{\ell}$ channel by just taking $\rho=0$. In terms of the variables $x_{ \pm}$, that corresponds to taking $x_{+}=1-r$ and $x_{-}=0$. We check our results by doing both, taking the massless limit and computing them from scratch by using the master integrals in Secs. III D and III E. They read

$$
\begin{gathered}
\mathcal{C}_{0}^{\mathrm{LO}}=48 \pi^{2}(1-r)^{2}(1+2 r), \\
\mathcal{C}_{0}^{\mathrm{NLO}}=-4 \pi^{2}\left[3(1-r)\left(6 r^{2}-9 r-5\right)\right. \\
+12 r(1+r)(1-2 r) \ln (r) \\
+6(1-r)^{2}(5+4 r) \ln (1-r) \\
\left.+12(1-r)^{2}(1+2 r)\left(\frac{\pi^{2}}{2}+\mathrm{Li}_{2}(r)-\mathrm{Li}_{2}(1-r)\right)\right] \\
=-4 \pi^{2}\left[3(1-r)\left(6 r^{2}-9 r-5\right)+12 r(1+r)(1-2 r) \ln (r)\right. \\
+6(1-r)^{2}(5+4 r) \ln (1-r) \\
\left.+24(1-r)^{2}(1+2 r)\left(2 \mathrm{~L}_{\mathrm{R}}(r)+\mathrm{L}_{\mathrm{R}}(1-r)\right)\right] .
\end{gathered}
$$

Note that the combination $\mathrm{Li}_{2}(r)-\mathrm{Li}_{2}(1-r)$ does not produce any factor of $\pi^{2}$ after integration, so to the total width. That means that the $\pi^{2}$ term in the coefficient of the total width comes only from the integration of the term with explicit $\pi^{2}$ in Eq. (60). In Eq. (61), the $\pi^{2}$ terms are completely hidden inside Roger's dilogarithms.

\section{B. Total (integrated) decay width}

Once the coefficient of the differential semileptonic decay width is obtained, we can compute the coefficient of the total semileptonic decay width as a check. It is obtained by solving the following integral:

$$
C_{0}(\rho)=\frac{1}{24 \pi^{2}} \int_{0}^{(1-\sqrt{\rho})^{2}} d r \mathcal{C}_{0}(r, \rho)
$$

Note that the expression above requires an integration over $r$. However, our expression for $\mathcal{C}_{0}(r, \rho)$ is written in terms of $x_{ \pm}(r, \rho)$, which are more natural variables for describing the system. As we previously discussed, these variables keep the expressions for the coefficients rather compact and in terms of simple structures which are polynomials in $x_{ \pm}$, or polynomials multiplied by logarithms, square logarithms, and dilogarithms. On the contrary, if we rewrite the coefficient of the differential width in terms of $r$ and $\rho$, the structure turns out to be much more complicated and integration becomes nonviable. Therefore, it is much better to integrate over $x_{+}$or $x_{-}$instead of $r$. First, we use $x_{+}=$ $\rho / x_{-}$in order to write $\mathcal{C}_{0}$ in terms of a single variable $x_{-}(\rho$ is constant). Then, $d r=\left(-1+\rho / x_{-}^{2}\right) d x_{-}$and the integral becomes 


$$
C_{0}(\rho)=\frac{1}{24 \pi^{2}} \int_{\sqrt{\rho}}^{\rho} d x_{-}\left(1-\frac{\rho}{x_{-}^{2}}\right) \mathcal{C}_{0}\left(x_{+}=\frac{\rho}{x_{-}}, x_{-}\right)
$$

After integration we find the coefficients for the $b \rightarrow c \ell \bar{\nu}_{\ell}$ channel

$$
\begin{aligned}
C_{0}^{\mathrm{LO}}=1-8 \rho+8 \rho^{3}-\rho^{4}-12 \rho^{2} \ln (\rho) \\
C_{0}^{\mathrm{NLO}}=\frac{25}{8}-\frac{\pi^{2}}{2}-\frac{239 \rho}{6}+16 \pi^{2} \rho^{3 / 2}-8 \pi^{2} \rho^{2}+16 \pi^{2} \rho^{5 / 2}+\frac{239 \rho^{3}}{6}-\frac{25 \rho^{4}}{8}-\frac{\pi^{2} \rho^{4}}{2} \\
+\frac{17}{6} \ln (1-\rho)+\frac{32}{3} \rho \ln (1-\rho)-\frac{32}{3} \rho^{3} \ln (1-\rho)+\frac{17}{6} \rho^{4} \ln (1-\rho) \\
-10 \rho \ln (\rho)-45 \rho^{2} \ln (\rho)+\frac{2}{3} \rho^{3} \ln (\rho)-\frac{17}{6} \rho^{4} \ln (\rho) \\
-32 \rho^{3 / 2} \ln (1-\sqrt{\rho}) \ln (\rho)-32 \rho^{5 / 2} \ln (1-\sqrt{\rho}) \ln (\rho) \\
+32 \rho^{3 / 2} \ln (1+\sqrt{\rho}) \ln (\rho)+32 \rho^{5 / 2} \ln (1+\sqrt{\rho}) \ln (\rho) \\
+2 \ln (1-\rho) \ln (\rho)+60 \rho^{2} \ln (1-\rho) \ln (\rho)+2 \rho^{4} \ln (1-\rho) \ln (\rho)-18 \rho^{2} \ln ^{2}(\rho)-\frac{1}{2} \rho^{4} \ln ^{2}(\rho) \\
+\left(6+64 \rho^{3 / 2}+96 \rho^{2}+64 \rho^{5 / 2}+6 \rho^{4}\right) \operatorname{Li}_{2}(-\sqrt{\rho}) \\
+\left(6-64 \rho^{3 / 2}+96 \rho^{2}-64 \rho^{5 / 2}+6 \rho^{4}\right) \operatorname{Li}_{2}(\sqrt{\rho}),
\end{aligned}
$$

which are in agreement with the previously known results given in Refs. [30,31,37]. Indeed, the expression above can be written more compactly by choosing a different set of dilogarithms to express the result

$$
\begin{aligned}
C_{0}^{\mathrm{NLO}}= & 128 \rho^{3 / 2}(\rho+1) \mathrm{Li}_{2}(1-\sqrt{\rho})-\left(32 \rho^{5 / 2}+32 \rho^{3 / 2}+3 \rho^{4}+48 \rho^{2}+3\right) \mathrm{Li}_{2}(1-\rho) \\
& -\frac{1}{2} \rho^{2}\left(\rho^{2}+36\right) \ln ^{2}(\rho)+\frac{1}{24}\left(-75 \rho^{4}+956 \rho^{3}-956 \rho+75\right) \\
& +\frac{1}{6}\left(17 \rho^{4}-64 \rho^{3}+64 \rho-17\right) \ln (1-\rho)-\left(\rho^{4}-12 \rho^{2}+1\right) \ln (1-\rho) \ln (\rho) \\
& -\frac{1}{6} \rho\left(17 \rho^{3}-4 \rho^{2}+270 \rho+60\right) \ln (\rho),
\end{aligned}
$$

or even in terms of Roger's dilogarithm

$$
\begin{aligned}
C_{0}^{\mathrm{NLO}}= & 128 \rho^{3 / 2}(\rho+1) \mathrm{L}_{\mathrm{R}}(1-\sqrt{\rho})-\left(32 \rho^{5 / 2}+32 \rho^{3 / 2}+3 \rho^{4}+48 \rho^{2}+3\right) \mathrm{L}_{\mathrm{R}}(1-\rho) \\
& -32 \rho^{3 / 2}(\rho+1) \ln (1-\sqrt{\rho}) \ln (\rho)-\frac{1}{2} \rho^{2}\left(\rho^{2}+36\right) \ln ^{2}(\rho) \\
& +\frac{1}{24}\left(-75 \rho^{4}+956 \rho^{3}-956 \rho+75\right)+\frac{1}{6}\left(17 \rho^{4}-64 \rho^{3}+64 \rho-17\right) \ln (1-\rho) \\
& -\frac{1}{6} \rho\left(17 \rho^{3}-4 \rho^{2}+270 \rho+60\right) \ln (\rho) \\
& +\frac{1}{2}\left(32 \rho^{5 / 2}+32 \rho^{3 / 2}+\rho^{4}+72 \rho^{2}+1\right) \ln (1-\rho) \ln (\rho) .
\end{aligned}
$$

Note that in both cases explicit $\pi^{2}$ terms are hidden inside dilogarithms.

For the $b \rightarrow u \ell \bar{\nu}_{\ell}$ channel, the integration of Eq. (63) over $r$ can easily be performed since $\rho=0$. Alternatively, we can take the massless limit in Eqs. (65)-(67). In both cases we obtain agreement with the known result

$$
C_{0}^{\mathrm{LO}}=1, \quad C_{0}^{\mathrm{NLO}}=\frac{1}{8}\left(25-4 \pi^{2}\right) .
$$




\section{CONCLUSIONS}

We have presented an alternative method for the computation of the decay width differential in the $n$ $p$-particle invariant mass square at NLO in the strong coupling $\alpha_{s}$, assuming a general transition $Q(M) \rightarrow$ $q(m) p_{1}\left(m_{1}\right) \cdots p_{n}\left(m_{n}\right)$. The approach is based on the use of the optical theorem, the HQE, and dispersion representations. The main result of the paper is the computation of the associated master integrals analytically, which are universal to this kind of processes. We have observed that the key point for the analytical computation of the master integrals in the massive case $(m \neq 0)$ is the choice of appropriate variables for the description of the system. We also compute the master integrals in the massless $(m=0)$ case as a check.

The coefficient of the differential width can always be computed analytically. Being able to get a completely analytical result or not will depend on the complexity of the spectral density $\rho_{s}$. If $\rho_{s}$ can be computed analytically, then the differential width is known analytically and the final integration over $r$ to obtain the total width or moments (with or without cuts) can always be performed, at least numerically, which is enough for comparison to experiment.

As an application, we have computed the leading HQE coefficient of the semileptonic decay width differential in the dilepton pair invariant mass square at NLO in $\alpha_{s}$. The decay is mediated by the $b \rightarrow q \ell \bar{\nu}_{\ell}$ transition, where the leptons are considered to be massless and $q$ can be either massive or massless. By a choice of variables, the differential width for the massive case can be expressed in terms of simple structures which are polynomials and polynomials multiplied by logarithms and Roger's dilogarithms (or alternatively polynomials multiplied by square logarithms and dilogarithms). The differential width for the massless case is computed as a check of our methods. We compute it explicitly and compare with the massive case in the limit $m \rightarrow 0$. As an additional check, we integrate the differential width to get the total semileptonic width and compare with the known result.

The master integrals we have computed may have several physical applications. They can be used for the computation of the differential and total semileptonic and nonleptonic decay widths with any number of different masses, or to topologically analogous decays emerging from new physics interactions.

The computation of the leptonic spectrum in $B$ or $D$ weak decays is the main application of our results. Still it would be interesting to explore if the method can also be applied to different kinds of weak decays currently studied in phenomenology such as $B \rightarrow X_{s} \ell^{+} \ell^{-}, B \rightarrow X_{s} \gamma$, or the decay of the top quark. The process $B \rightarrow s \mu^{+} \mu^{-}$has drawn a lot of attention recently in relation to the observation of flavor anomalies. For reliable conclusions about the deviations from the SM, a high accuracy of theoretical predictions is required, which also includes an account of subleading terms in the large $m_{b}$ power expansion. To the best of our knowledge, the NLO contributions of the operators $O_{9}$ and $O_{10}$ of the effective Lagrangian (see e.g., [38]) to the differential rate are unknown at present, and can readily be computed using the master integrals presented in this paper. The same applies to $B \rightarrow s \tau^{+} \tau^{-}$, where the $\tau$ mass can readily be accounted in our setup. The NLO contribution of the operator $O_{7}$ to the total rate of $B \rightarrow X_{s} \gamma$ can also be computed within our approach though it was obtained earlier within different methods.

Another potential application is the top quark decay into $b$-quark and $W$-boson. The state of the art is the evaluation of the decay rate at NNLO for a massless $b$-quark with some expansion in the $W$-boson mass $[39,40]$. The master integrals of our paper can be used to compute the rate at NLO for a massive $b$ quark. Even though at this level the results are already known, the techniques presented in the paper are somewhat simpler than direct computations of the decay width.

Finally, the master integrals obtained in the paper can appear as a part of the computation of more complicated processes with a larger number of loops and/or in the higher orders of mass expansions.

As an immediate application of the master integrals presented here we have computed analytically the $1 / \mathrm{m}_{b}^{3}$ power corrections to the HQE of the differential rate of $B \rightarrow X_{c} \ell \bar{\nu}$ at NLO-QCD [41].

\section{ACKNOWLEDGMENTS}

This research was supported by the Deutsche Forschungsgemeinschaft (DFG, German Research Foundation) under Grant No. 396021762 - TRR 257 "Particle Physics Phenomenology after the Higgs Discovery."

\section{APPENDIX: DISPERSION REPRESENTATION OF THE ONE-LOOP TWO-PROPAGATOR DIAGRAM}

Two-point functions obey dispersion relations which follow from the analyticity properties of these functions $[42,43]$. In particular, the dispersion representation of the one-loop diagram with two propagators plays a key role in this work. It is not only extremely useful for the computation of master integrals, but most importantly it allows one to write the expression for the semileptonic width in such a way that the decay width differential in the leptonic pair invariant mass can be identified. For this reason, we present it here explicitly due to its relevance to the paper.

In the case of two massive lines with different masses the dispersion representation of the one-loop sunset reads 


$$
\frac{1}{i} \int \frac{d^{D} q}{(2 \pi)^{D}} \frac{1}{q^{2}-m_{1}^{2}} \frac{1}{(p-q)^{2}-m_{2}^{2}}=\int_{\left(m_{1}+m_{2}\right)^{2}}^{\infty} \frac{\rho_{1 \mathrm{LS}}\left(\xi, m_{1}, m_{2}\right)}{\xi-p^{2}-i \eta} d \xi
$$

where

$$
\rho_{1 \mathrm{LS}}\left(\xi, m_{1}, m_{2}\right)=\frac{1}{(4 \pi)^{\lambda+1}} \frac{\Gamma(\lambda)}{\Gamma(2 \lambda)} \xi^{\lambda-1}\left[\left(1-\frac{\left(m_{1}+m_{2}\right)^{2}}{\xi}\right)\left(1-\frac{\left(m_{1}-m_{2}\right)^{2}}{\xi}\right)\right]^{\lambda-1 / 2}
$$

with $D=2 \lambda+2=4-2 \epsilon$, is the spectral density.

[1] J. Chay, H. Georgi, and B. Grinstein, Phys. Lett. B 247, 399 (1990).

[2] I. I. Y. Bigi, N. G. Uraltsev, and A. I. Vainshtein, Phys. Lett. B 293, 430 (1992); 297, 477(E) (1992).

[3] A. V. Manohar and M. B. Wise, Phys. Rev. D 49, 1310 (1994).

[4] B. Blok, L. Koyrakh, M. A. Shifman, and A. I. Vainshtein, Phys. Rev. D 49, 3356 (1994); 50, 3572(E) (1994).

[5] T. Mannel, Nucl. Phys. B413, 396 (1994).

[6] A. Czarnecki and M. Jezabek, Nucl. Phys. B427, 3 (1994).

[7] M. B. Voloshin, Phys. Rev. D 51, 4934 (1995).

[8] A. F. Falk, M. E. Luke, and M. J. Savage, Phys. Rev. D 53, 2491 (1996).

[9] C. W. Bauer and B. Grinstein, Phys. Rev. D 68, 054002 (2003).

[10] M. Trott, Phys. Rev. D 70, 073003 (2004).

[11] N. Uraltsev, Int. J. Mod. Phys. A 20, 2099 (2005).

[12] V. Aquila, P. Gambino, G. Ridolfi, and N. Uraltsev, Nucl. Phys. B719, 77 (2005).

[13] P. Gambino, P. Giordano, G. Ossola, and N. Uraltsev, J. High Energy Phys. 10 (2007) 058.

[14] B. Capdevila, P. Gambino, and S. Nandi, J. High Energy Phys. 04 (2021) 137.

[15] R. Lee, arXiv:1212.2685.

[16] R. N. Lee, J. Phys. Conf. Ser. 523, 012059 (2014).

[17] Q. Ho-kim and X. Y. Pham, Ann. Phys. (N.Y.) 155, 202 (1984).

[18] G. 't Hooft and M. J. G. Veltman, Nucl. Phys. B44, 189 (1972).

[19] K. G. Chetyrkin, M. Misiak, and M. Munz, Nucl. Phys. B520, 279 (1998).

[20] A. G. Grozin, T. Mannel, and A. A. Pivovarov, Phys. Rev. D 96, 074032 (2017).

[21] G. Altarelli, G. Curci, G. Martinelli, and S. Petrarca, Nucl. Phys. B187, 461 (1981).

[22] A. J. Buras and P. H. Weisz, Nucl. Phys. B333, 66 (1990).
[23] A. J. Buras, M. Jamin, and P. H. Weisz, Nucl. Phys. B347, 491 (1990).

[24] M. S. Chanowitz, M. Furman, and I. Hinchliffe, Nucl. Phys. B159, 225 (1979).

[25] M. Jamin and M. E. Lautenbacher, Comput. Phys. Commun. 74, 265 (1993).

[26] T. Huber and D. Maitre, Comput. Phys. Commun. 175, 122 (2006).

[27] T. Huber and D. Maitre, Comput. Phys. Commun. 178, 755 (2008).

[28] S. Narison and A. A. Pivovarov, Phys. Lett. B 327, 341 (1994).

[29] M. Fael, T. Mannel, and K. Keri Vos, J. High Energy Phys. 02 (2019) 177.

[30] T. Mannel, A. A. Pivovarov, and D. Rosenthal, Phys. Lett. B 741, 290 (2015).

[31] T. Mannel, A. A. Pivovarov, and D. Rosenthal, Phys. Rev. D 92, 054025 (2015).

[32] L. Adams, C. Bogner, and S. Weinzierl, Proc. Sci., RADCOR2015 (2016) 096 [arXiv:1601.03646].

[33] A. N. Kirillov, Prog. Theor. Phys. Suppl. 118, 61 (1995).

[34] G. Bell, M. Beneke, T. Huber, and X. Q. Li, Nucl. Phys. B843, 143 (2011).

[35] G. Buchalla, A. J. Buras, and M. E. Lautenbacher, Rev. Mod. Phys. 68, 1125 (1996).

[36] A. Grozin, arXiv:hep-ph/0508242.

[37] Y. Nir, Phys. Lett. B 221, 184 (1989).

[38] A. Ali, G. Hiller, L. T. Handoko, and T. Morozumi, Phys. Rev. D 55, 4105 (1997).

[39] I. R. Blokland, A. Czarnecki, M. Slusarczyk, and F. Tkachov, Phys. Rev. Lett. 93, 062001 (2004).

[40] I. R. Blokland, A. Czarnecki, M. Slusarczyk, and F. Tkachov, Phys. Rev. D 71, 054004 (2005); 79, 019901(E) (2009).

[41] T. Mannel, D. Moreno, and A. A. Pivovarov, arXiv:2112 .03875 .

[42] G. Kallen, Helv. Phys. Acta 25, 417 (1952).

[43] H. Lehmann, Nuovo Cimento 11, 342 (1954). 